\title{
Carbon nanotube: Controlled synthesis determines its future
}

\author{
Shuchen Zhang ${ }^{1}$, Liu Qian ${ }^{1}$, Qiuchen Zhao ${ }^{1}$, Zequn Wang ${ }^{1}$, Dewu Lin ${ }^{1}$, Weiming Liu ${ }^{1}$, \\ Yabin Chen ${ }^{2}$ and Jin Zhang ${ }^{1 *}$
}

\begin{abstract}
Carbon nanotubes (CNTs) have received broad attention in the past decades due to their excellent physical and chemical properties and thus been regarded as a powerful candidate for future star-materials. Although various CNT products and their related applications have been demonstrated recently, their performance can hardly meet the researchers' expectations compared with their theoretical properties. The current predicament is caused by the immature synthesis method, including the basic science and the producing technology. As the synthesis with controlled structures determines its future, this review summarizes the progress on the basic research and industrialization of CNTs in the past decades, including the fine structure control, aggregation status design and scale-up production, and further points out the way for the future development of CNTs combining with specific applications.
\end{abstract}

Keywords: carbon nanotubes, structure-controlled synthesis, scale-up synthesis, applications

\section{INTRODUCTION}

Carbon nanotubes (CNTs) discovered in 1991 [1], possess the unique structure and superior properties as a typical one-dimensional (1D) nanomaterial. Since single-walled carbon nanotube (SWNT) was reported in 1993 [2], various topological structures, caused by rolling up graphene nanosheets with different sizes along different directions, provide much more fantastic properties in nanoelectronics [3-5] and nano-photonics [6-8]. Specifically, SWNTs could be either metallic (m-SWNT) or semiconducting (s-SWNT) depending on their topological structures [9]. Due to the advantages of high tensile strength [10], high carrier mobility [11], and large specific surface area [12], CNTs have already shown broad ap- plications [13], including high-performance nano-electronic devices, sensors and energy conversion devices. At present, the worldwide commercial interest in CNTs is the production capacity of several thousand tons per year [14]. However, optional use of CNTs in numerous fields led to an embarrassing situation for CNT and it is necessary to reassess the structure, properties and related applications of CNTs before the upcoming industrialization.

Synthesis determines the future. To make the best use of the diverse advantages of CNTs towards applications, many researchers focused on their synthesis in the past decades. Throughout the development of CNTs synthetic technology, two obvious trends are the fine structure control of CNTs [15-17], especially SWNTs, and the aggregation and scale-up synthesis [18-20]. Although great achievements have been made, the gap between the fine-structure control and scale-up synthesis seems to become larger. In other words, it is hard to combine quantity (increasing yield and reducing cost) and highquality (improving purity and selectivity) in CNT production, which hinders further popularization. Which kind of CNTs benefits the specific application considering the balance between performance and cost? How to highlight these properties of CNTs from the view of synthesis? Before answering these questions, the era of CNTs would never come. In this review, we briefly summarize the progress of basic research and industrialization of CNTs in the past decades, and try to point out the direction for the future development of CNTs. The current application field of CNTs and related requirements for further applications are also introduced. Based on these requirements, an overview on CNT synthesis from the aspects of fine structure control, ag-

\footnotetext{
${ }^{1}$ Center for Nanochemistry, Beijing Science and Engineering Center for Nanocarbons, Beijing National Laboratory for Molecular Sciences, College of Chemistry and Molecular Engineering, Peking University, Beijing 100871, China

${ }^{2}$ School of Aerospace Engineering, Beijing Institute of Technology, Beijing 100081, China

* Corresponding author (email: jinzhang@pku.edu.cn)
} 
gregation form control and scale-up synthesis is made. Finally, we give a perspective on each section, and a blueprint for the development of CNTs in the future.

\section{STRUCTURES AND PROPERTIES OF CNTs}

\section{Geometry and electronic structure}

CNT could be regarded as a seamless cylinder rolled up by one or multi-layers of graphene [1,2]. Each CNT is composed of three parts as cap, wall and edge, as shown in Fig. 1a. In view of bonding, the central wall of a perfect CNT has all carbon atoms bonded in a hexagonal lattice and each atom connects with three nearest ones via $\mathrm{sp}^{2}$ hybridized orbitals, leaving the $\pi$ electron delocalized over the whole sidewall. In comparison, the fine structure of cap remains unclear, because of the various possibilities with pentagon or heptagon defects. It is believed that the cap is the main decisive factor of the unique structure of CNTs, while the original edge connected to the catalyst is actually the true executor causing its chiral angle [21]. The bare edges without any catalysts could be created by cutting a CNT while these edges could be rapidly functionalized when exposed in air [22]. As a typical 1D nanomaterial, CNT displays the superior length/diameter ratio. The diameter usually ranges from 0.8 to $2.0 \mathrm{~nm}$ for SWNTs, and up to several hundred nan- ometers for multi-walled carbon nanotube (MWNTs) [14], with the length exceeding half a meter [23], which makes CNTs like super molecules or single crystals.

Although there is a structural similarity to graphene, CNTs, especially SWNTs, present very fantastic electronic structures by introducing different curling vectors to the graphene sheets, which makes the SWNTs to be either semiconducting or metallic. The electrical behavior of a given SWNT could be simply distinguished from its chiral index $(n, m)$ [24]. If $n-m=3 k$ ( $k$ is an integer), the SWNTs are metallic (only for armchair types, $k=0$ ) or semi-metallic with a tiny band gap, otherwise they exhibit obvious semiconducting behavior with a sizeable band gap inversely proportional to the diameter. For example, $(19,0)$ nanotube is semiconducting and its electronic structure is shown in Fig. 1b [6]. Generally, MWNTs are metallic and usually present the similar electrical properties to those of metallic SWNTs. Moreover, MWNTs and metallic SWNTs can achieve the ballistic transport over a long mean free path and thus can carry an impressive current density. For example, MWNTs can load a current up to $10^{9} \mathrm{~A} \mathrm{~cm}^{-2}$, much higher than mixed SWNTs [25].

\section{Excellent properties decide the wide applications}

The excellent geometrical and electrical properties of
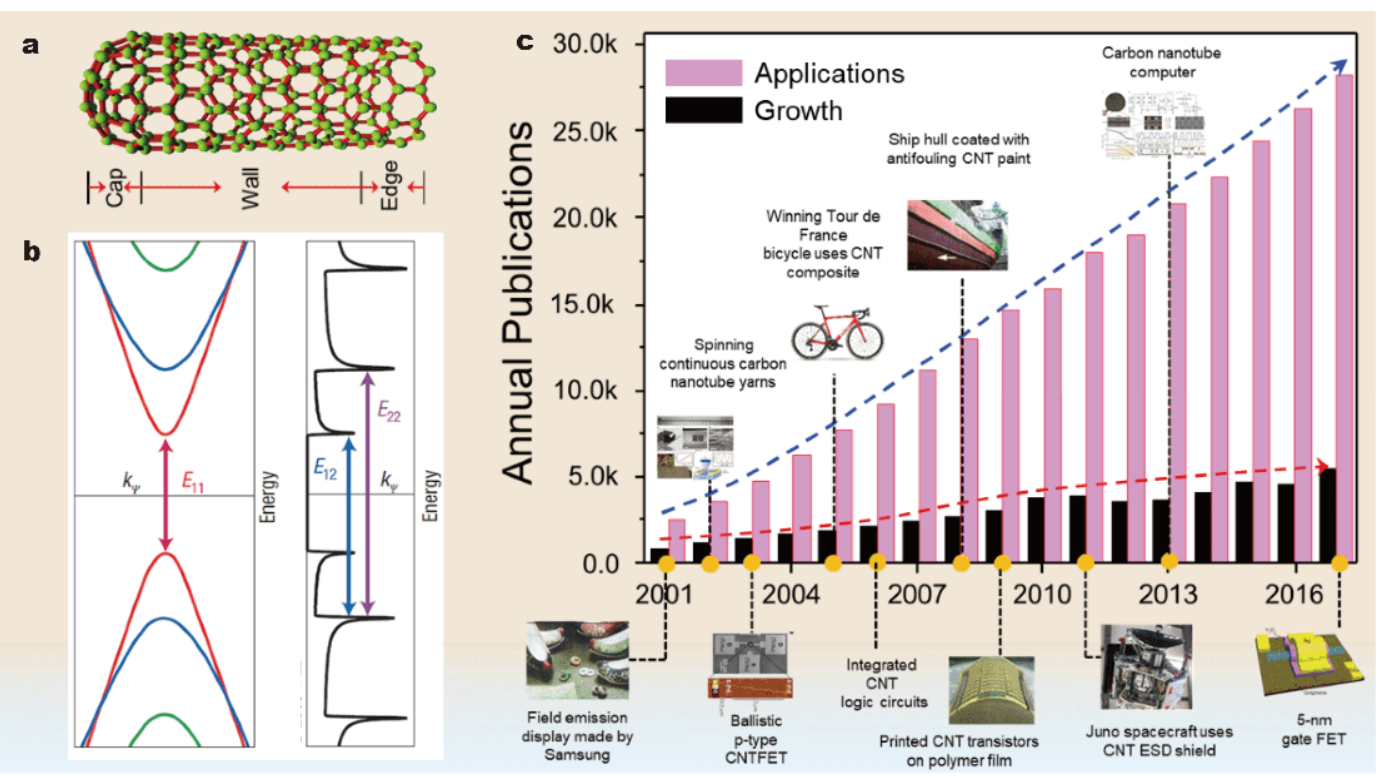

Figure 1 Structure of CNTs decides excellent properties, which further decides the wide applications. Geometric structure (a) of CNT and electronic structure (b) of $(19,0)$ nanotube, which indicate its hollow cylinder structure and the semiconducting property (Reprinted with permission from Ref. [6], Copyright 2008, Springer Nature). (c) Journal publications in Web of Science according to the keywords "carbon nanotubes, applications" and "carbon nanotubes, growth", respectively. Many applications have been explored, which is reflected by the great increase in the number of relative publications and structure-controlled growth of CNTs is still a big challenge with a slow growth of the related publications. 
CNTs determine their various applications in different fields. As shown in Fig. 1c, the annual number of CNTrelated publications continues to grow. Because of the stable and strong carbon-carbon covalent bond, CNTs possess the similar elastic modulus and classy tensile strength with graphene. For example, the elastic modulus and tensile strength of an individual MWNT are $1 \mathrm{TPa}$ and $100 \mathrm{GPa}$, respectively, which are over 10 -fold stronger than carbon fiber [10]. Therefore, CNTs can be used as additive to enhance the strength of other materials, such as polymers or precursor resins [26]. More and more CNT-based composites have been successfully applied into our daily supplies, such as bicycles and badminton rackets [14].

Ultra-high electron and hole mobilities further prove the excellent electrical property of CNTs, both up to $100,000 \mathrm{~cm}^{2} \mathrm{~V}^{-1} \mathrm{~s}^{-1}$ in theory [27]. Therefore, MWNTs or SWNTs without any post-growth treatment are often directly used as coatings, films or guest in composites to enhance the conductivity of the host materials, and improve the electron transport for energy storage. For example, the improved conductivities of $10,000 \mathrm{~S} \mathrm{~m}^{-1}$ have been realized for polymethyl methacrylate (PMMA) when containing $10 \mathrm{wt} \% \mathrm{SOCl}_{2}$ treated SWNTs [28], and of $2000 \mathrm{~S} \mathrm{~m}^{-1}$ for polyurethane (PU) with $15 \mathrm{wt} \%$ MWNTs [29]. Besides the metallic SWNTs, $2 / 3$ of SWNTs are semiconducting with proper band gaps, which are considered as the most promising candidates to replace silicon in semiconductor industry. At present, plenty of CNT-based field effect transistors (FETs) [4,30-32] and thin film transistors (TFTs) $[33,34]$ have been obtained in the laboratory. For the lower scattering rate and smaller effective mass of electrons, CNTs are more competitive than other materials for high-performance transistor devices.

Except for the mechanical and electrical properties, the unique structures of CNTs also favor the phonons to propagate along the nanotube smoothly. Thermal conductivity of an individual CNT is about $3000 \mathrm{~W} \mathrm{mK}^{-1}$ for MWNTs [35], and up to $3500 \mathrm{~W} \mathrm{mK}^{-1}$ for SWNTs [36], which are comparable to those of other carbon allotropies, diamond and graphite. In fact, the experimental measurements on thermal conductivity of CNTs usually show the significantly scattered results, in the range of 2000 to $6000 \mathrm{~W} \mathrm{mK}^{-1}$, far behind the simulated results [37]. A series of applications have been developed based on the high thermal conductivity of CNTs, such as polymer/CNTs nanocomposites or coatings. Thermal conductivity of MWNTs-filled polypropylene composites could be improved by $\sim 3$ times compared with pure polypropylene matrix $\left(\sim 0.6 \mathrm{~W} \mathrm{mK}^{-1}\right)$ [38]. CNTs composites can also be used as the main building blocks for thermal management. When $1 \mathrm{wt} \%$ SWNT powder was added to the industrial epoxy, the thermal conductivity increased $70 \%$ at $40 \mathrm{~K}$, and up to $125 \%$ at room temperature [39].

CNTs also exhibit a high chemical stability and good chemical compatibility with biomolecules due to the carbon-carbon bond, such as DNA and proteins [14]. Therefore, they can play a vital role in bio-imaging, biosensors, or environment monitoring. In bio-imaging, SWNTs are preponderantly applied as a local heater in near-infrared radiation [40], photoacoustic imaging [41], and fluorescent [42]. For CNT biosensors, they can sensitively response to the surrounding change of biological internal environment through recording the electrical impedance or optical properties. For the environmentrelated application, especially water purification for commercialization, tangle CNTs were fabricated into porous filters with controlled size, and then used to electrochemically remove organic contaminants, bacteria, and viruses [43-45].

Although many applications have been explored, unfortunately most of them could not be converted into the commercial products, because they usually show the excellent performance on single property, but could not meet the stringent requirement of the market. In order to solve this issue, a killer application combining various advantages of CNTs must be focused on making a breakthrough for the life and science. A typical example is the CNT-FET, which requires both the high carrier mobility and the high thermal conductivity to ensure the heat dissipation for the devices.

\section{CONTROLLED SYNTHESIS DETERMINES THE FUTURE}

Synthesis determines the future of materials. To maximize the advantages of CNTs, many studies focused on the controlled synthesis in the last decades. As shown in Fig. 1c, the publication number related to the controlledgrowth of CNTs increased monotonically. In this section, the determinants for the controlled growth are analyzed, and then the representative studies are discussed as four aspects: geometric structure, electrical properties, aggregation, and scale-up growth. Finally, to better show the importance of controlled-growth, the corresponding applications related to each section are further illustrated.

\section{Applications need CNTs with controlled structures}

CNTs possess prominent properties. However, it is a pity 
that CNTs show unsatisfactory results in the practical applications for various reasons, including the structure and aggregates morphology. Therefore, structures of CNTs should be controlled by the specific design.

In practical applications, the key problem is that the individual CNT usually exhibits better properties than CNT bundle. The main reason is that multi CNTs with uniform structures have not been obtained till now. For example, CNTs in crude powder samples mixed with metal catalysts and excess amorphous carbon, inevitably have many defects, including lattice vacancy and pentagon-heptagon pair [46]. Another problem for CNTs samples is a wide distribution in length and diameter. For the growth of vertical CNT array, the length (or height) is an important criterion to evaluate the quality of the obtained array. Meanwhile, it is important to control the diameter and the wall number of CNTs to improve their properties. In addition to structure control, property control is also extremely important. For example, most MWNTs are metallic, but for SWNTs, 2/3 of them are semiconducting and the rest are metallic. It is a great challenge to separate these two types of SWNTs because of their structural similarity. For the solution-based separation methods, some pollutants are unavoidably introduced $[47,48]$ and would weaken the performance of CNTs [49], so to in-situ control the electrical property of CNTs during the growth is highly valued. In addition, another issue to grow SWNT array with uniform chirality is still unresolved till now.

For most of the practical applications, the aggregated CNTs are often necessary rather than the individual ones. Therefore, it is very important to control the properties and morphologies of CNTs aggregation. According to their morphology difference, CNT aggregates could be divided into three categories as $1 \mathrm{D}$ like high strength CNT fibers [50], 2D like CNT films for fabricating TFT or conductive film [33,51], and 3D like CNT sponge [20] and vertical array [52]. Without a doubt, multi-designs on dimensions greatly expand the application fields and increase their market competitiveness. In the near future, we believe that CNT slurry could be utilized in $3 \mathrm{D}$ printing technology to fabricate the products with desired morphology.

The controlled growth of CNTs not only lies in pursuing specific structure and properties, but also involves the corresponding products or applications. For any application, CNT aggregates could be realized by different methods, definitely leading to different performance. CNT material is usually evaluated based on the test results of the performance. Therefore, the material selection for application is blind and lacks an accepted standard to prior estimate these results. Therefore, it is fundamentally necessary to establish the industry standards for CNT products as well as carbon fibers. Taking CNT film as an example, growth method, length and diameter distribution, and impurity concentration should be fully listed in the reference standard. For CNT horizontal array, density is another important index.

\section{Geometry control improves the applications based on mechanical properties}

Geometric structures of CNTs mainly refer to the diameter (number of walls for MWNTs), length, defect and chirality. To control the geometric structure of CNTs, the key points include the connection between catalyst and CNTs, temperature, and gas atmosphere, of which, the catalyst plays the essential role to modulate the fine structure of the grown CNTs by changing its state, activity and composition.

\section{Diameter control}

It is generally believed that the diameter of CNT is mainly associated with the size of catalyst both for SWNTs and MWNTs [53], which has been proved by the TEM results in Fig. 2a [54-56]. Besides diameter, the catalyst size also affects the number of walls for MWNTs. The statistics in Fig. 2b show the relationship between CNT diameter and the size of catalyst. It can be concluded that catalyst particles with less than $5 \mathrm{~nm}$ mainly produce SWNTs, and the ones larger than $10 \mathrm{~nm}$ mainly produce MWNTs. For the size between 5 and $10 \mathrm{~nm}$, both SWNTs and MWNTs can be synthesized. The final results depend on the growth condition. Generally, it tends to produce SWNTs with etching gas and MWNTs with excess carbon, respectively.

For MWNTs, it is much more meaningful to control the number of walls than the diameter, and it can be realized by introducing etching gas during the growth $[57,58]$. Typically, water is a good choice and the wall number of CNTs can be tuned by the partial pressure of water. Based on this principle, vertical array with highly pure SWNTs was realized [59]. For SWNTs, much attention has been paid to the diameter control, in order to explore the limited diameters, especially the smallest ones [60] and to get SWNTs with designated diameter. The former is based on the curiosity of science, and the latter is more valuable in practical application. Great efforts have been made to control the CNT diameter by designing catalysts. As a result, how to get a narrow size distribution of metal or alloy nanoparticles is the primary 

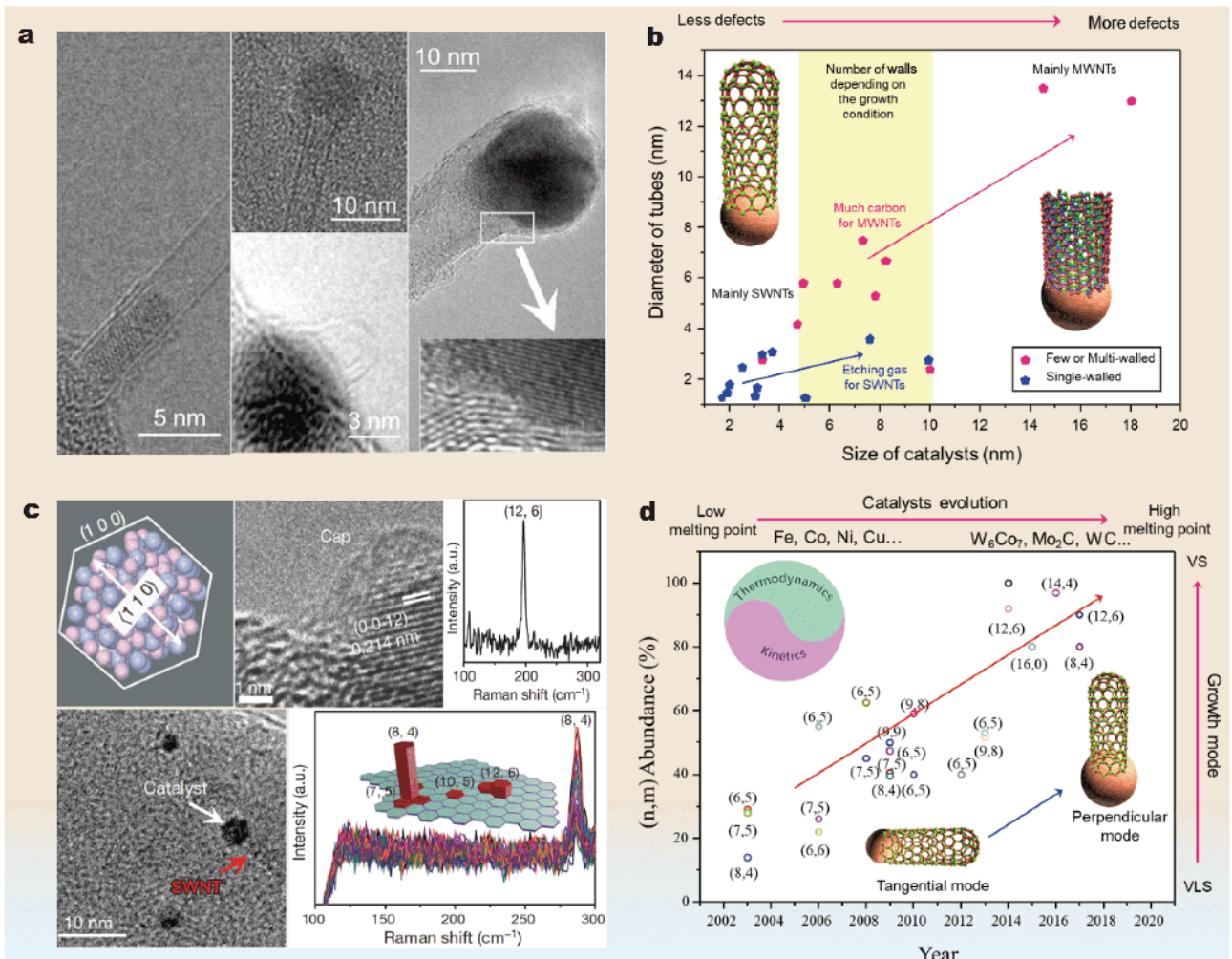

Figure 2 Geometry structure control of CNTs. (a) Catalysts with different sizes to control the CNTs diameter or the number of walls. (Reprinted with permission from Ref. [54], Copyright 2017, Elsevier; [55], Copyright 2008, Elsevier; [56], Copyright 2012, American Chemical Society). (b) The relationship between the size of catalysts and diameter of CNTs: small catalysts for SWNTs and big catalysts for MWNTs. (c) Solid catalysts to realize the chirality control. (Reprinted with permission from Ref. [65], Copyright 2014, Springer Nature; [70], Copyright 2017, Springer Nature). (d) Developing trend in the chirality control (Reprinted with permission from Ref. [71], Copyright 2017, Oxford University Press).

challenge. At the early stage, controlling the size and structure of catalyst precursor is considered as an effective way. By methods like importing a bit of iron into the cores of apoferritin 61 or controlling the size of nearly monodispersed nanoclusters $[53,62]$, the target size distribution of catalyst precursor is achieved. However, even the structure of catalyst precursor can be designed, its real size can still vary at high temperature through agglomeration, evaporation or Ostwald ripening, especially for the catalysts with low melting point which are often considered as liquid state during the growth [63]. Thus, it is not enough to simply control the structure of precursor, and the good stability of catalyst nanoparticles at high temperature is also important. An effective way is to use solid catalyst, such as $\mathrm{TiO}_{2}$ [64], W/Co alloy [64,65], and $\mathrm{Mo}_{2} \mathrm{C}$ [66]. Some of them can even keep a specific crystal face under high temperature, which is good for the growth of specific SWNTs at high temperature. However, even if the size distribution of solid catalysts is controlled to be extremely narrow, the size distribution of SWNTs still exists and may be caused by the different contact modes between SWNTs and catalysts.

In recent years, studies on simply controlling the CNT diameter have been reduced gradually, because it cannot determine its properties eventually. SWNTs with similar diameter can present completely different electrical properties. However, controlling the chirality of SWNT can determine its diameter at the same time. In recent publications, catalysts with high melting point are also suitable for the chirality control of SWNTs.

\section{Length control}

The length of the CNTs usually depends on their growth methods, typically $50-300 \mu \mathrm{m}$ for horizontal array $[64,66]$ and up to centimeter for vertical array [59]. But for powder samples, the length is only tens of microns because of the blocks formed by the supports [58]. Nevertheless, super-long CNTs have been put forward and a new strategy is proposed to enrich SWNTs with single chirality. The growth of super-long CNTs follows the "kite-flying" model [67], in which the catalysts detach from the substrate and come into the gas because of the 
thermal buoyancy. To obtain a much longer length, catalyst must keep its activity for a longer time. Super-long CNTs with $0.5 \mathrm{~m}$ length have been realized through moving flat-temperature zone [23]. Detailed analysis indicates that the length distribution of super-long CNTs conforms to Schulz-Flory distribution firstly used in polymer science, and the catalyst activity probability $(\alpha)$ can be evaluated. In the case of half-meter CNT, up to 0.995 of $\alpha$ means that the catalyst deactivation probability for adding one carbon dimer is lowered to $8.35 \times 10^{-11}$. Although synthesis of super-long CNTs paves the new way to enrich CNTs with uniform chirality, the yield is much limited.

\section{Chirality control}

The excellent properties of SWNTs arise from the topological structure, i.e., chirality. Chirality control is only involved in the growth of SWNTs. Realizing the precise control of SWNT chirality can solve most of the problems in the synthesis of individual nanotube. Therefore, chirality control is regarded as the "Holy Grail" in SWNTs synthesis. From the view of geometry point, the key to control the chirality is to control the formation of the hemispherical cap composed of six pentagons, which normally forms at the initial phase of SWNT growth. Recent publications mainly focus on how to control the cap or seed structure.

The seed growth, or "cloning" invented by Smalley et al. $[67,68]$, refers to the novel method which elongates SWNT from an opening end, and the newly added carbon-carbon six-membered ring can maintain the initial chirality of the SWNT seed $[22,69]$. Although this method is hopeful to obtain the SWNTs with nearly totally identical chirality, the growth efficiency strongly restricts its further improvement. Compared with carbon seeds, catalysts with high efficiency could be easily obtained in catalytic chemical vapor deposition (CVD) method. Catalyst is believed to be the most important factor affecting the chirality. As shown in Fig. $2 c$, since a rigid catalyst is beneficial for the chirality control of SWNTs, many solid catalysts, such as W/Co alloy [65], $\mathrm{Mo}_{2} \mathrm{C}$ and WC [70], have been developed to replace the traditional liquid catalysts. Fig. 2d indicates that the corresponding growth mechanism transfers from vapor-liquid-solid to vapor-solid-solid [71]. Obviously, the change of catalyst state improves the chirality selectivity of SWNTs.

Based on the solid catalysts $[65,70,72]$, the nucleation and growth of CNTs can be analyzed in the view of thermodynamics or kinetics. In thermodynamics, the chirality was defined by the composition and structure of solid catalysts, akin to the growth phenomenon between heterogeneous crystals. Then symmetry matching was put forward to control the chirality of CNTs, so $(12,6)$ and $(8$, 4) nanotubes were successfully enriched with high selectivity [70]. More importantly, this strategy can enrich one type of SWNTs but not limited to only one. A recent theory describes the nucleation thermodynamics by introducing the configurational entropy, and the chirality distribution under different temperatures combining with calculations is also demonstrated [73]. For these theories, much more experiments should be attempted to prove the exact nucleation thermodynamics of CNTs. On the other hand, the kinetic growth behavior was described using screw-dislocation theory [74], which pointed out that the growth rate depended on the number of dislocations or kinks. Specific kinetic growth behavior was speculated to be related to the growth modes depending on different catalysts $[74,75]$. Theoretically, two different modes of sphere and slab are widely used to study the growth dynamics of SWNT, which show different relationship between the growth rate and chiral angle. Experimentally, these two growth rate laws match well with two specific growth modes, named tangential and perpendicular modes (Fig. 2d) [54]. In tangential mode, the catalysts seem to be "soft" to own the same diameter with the nanotubes, and part of the catalyst atoms could be sucked into the nanotube. In perpendicular mode, however, the catalysts seem to be "hard" and stay out of the nanotubes with a larger size than the nanotubes. Both two modes exist in liquid catalyst system with only vertical growth mode for the solid catalyst system. As the growth mode can strongly affect growth preferences, to maintain a unique mode is beneficial for improving the selectivity.

In a word, it is vitally important to find or design the solid catalysts and to further build an exact relationship between the nanotubes and the active lattice plane of catalysts. It is well known that many common catalysts tend to be solid under a low growth temperature, and this strategy is usually adopted to synthesis powder sample with a slight selectivity of near-armchair nanotubes $[57,71]$. Though catalyst control is still an obstacle for controlling the SWNTs chirality, the selectivity can be further improved through deep understanding of catalysts in atomic scale.

\section{Defect control}

Defect is a serious problem for most materials, but not for CNTs because of the strong carbon-carbon covalent bond. It is hard to produce defects in CNTs, especially SWNTs at high growth temperature. Relevant theoretical 
calculations show that self-repairing on CNTs can happen quickly to make defects disappear at high temperature even if these defects have already formed [76]. Experiments indicate nearly no defects on SWNTs [77] and some defects on MWNTs [78]. The growth of MWNTs needs much more carbon source increasing the possibility to produce defects. Luckily, CNTs with perfect structure are pursued in most experiments and practical applications. Besides the intrinsic defects, the intentional doping is necessary for CNTs to adjust their properties, such as $\mathrm{N}$-doping [79] or carboxylation [80] to increase the solubility of CNTs in water. Chemical modifications have been developed mainly for MWNTs rather than SWNTs because this method can seriously break the wall structures of CNTs and form graphene nanoribbon or similar fragments [81]. At present, very little study has been done on the defects of CNTs. If the effective defect control can be realized, the application fields of CNTs could be broadened absolutely.

\section{Applications based on mechanical properties}

Based on the geometric structure of CNTs, two related applications have been developed, material synthesis and load-bearing applications $[26,82]$. For the former one, improving the structure control has a significant effect on the properties of CNT aggregates. Typically, through controlling diameter of CNTs in the vertical array, superaligned arrays formed and were directly fabricated into CNT fibers or films, as shown in Fig. 3a $[58,59,83]$. At present, super-aligned MWNT arrays have been realized in laboratory, but super-aligned SWNT arrays are rarely reported because diameter distribution has stronger effect on SWNTs than that on MWNTs. Improving diameter control of SWNTs to obtain super-aligned SWNT arrays with good spinning ability is still a great challenge.

For load-bearing applications, super strong CNT fibers or yarns are mainly fabricated to give full play to their excellent elastic modulus and high tensile strength, as shown in Fig. 3b [84]. Compared with some metal materials, CNT fibers show much higher tensile strength. CNT yarns made by high-quality few-walled CNTs have presented a strength of $8.8 \mathrm{GPa}$, which is still much less than that of the individual CNT [50]. Recent research shows that a possible reason is Daniels effect owing to the discontinuous lengths of CNTs. It means that not all CNTs work in testing situation. A synchronous tightening and relaxing strategy was developed to realize the uniform length of CNTs, and the measured tensile strength was as high as $80 \mathrm{GPa}$ [85]. In applications based on strength property, there is no obvious difference between MWNTs and SWNTs. Considering the low price of MWNTs, they are widely used in many fields, such as an additive to improve the strength of composites. In contrast, SWNTs is light in weight but with high cost, and a less amount of addition can greatly improve the strength of composites, so they are appropriate in many high-end applications.

\section{Band structure decides electrical applications}

As mentioned above, CNTs have two types of electrical
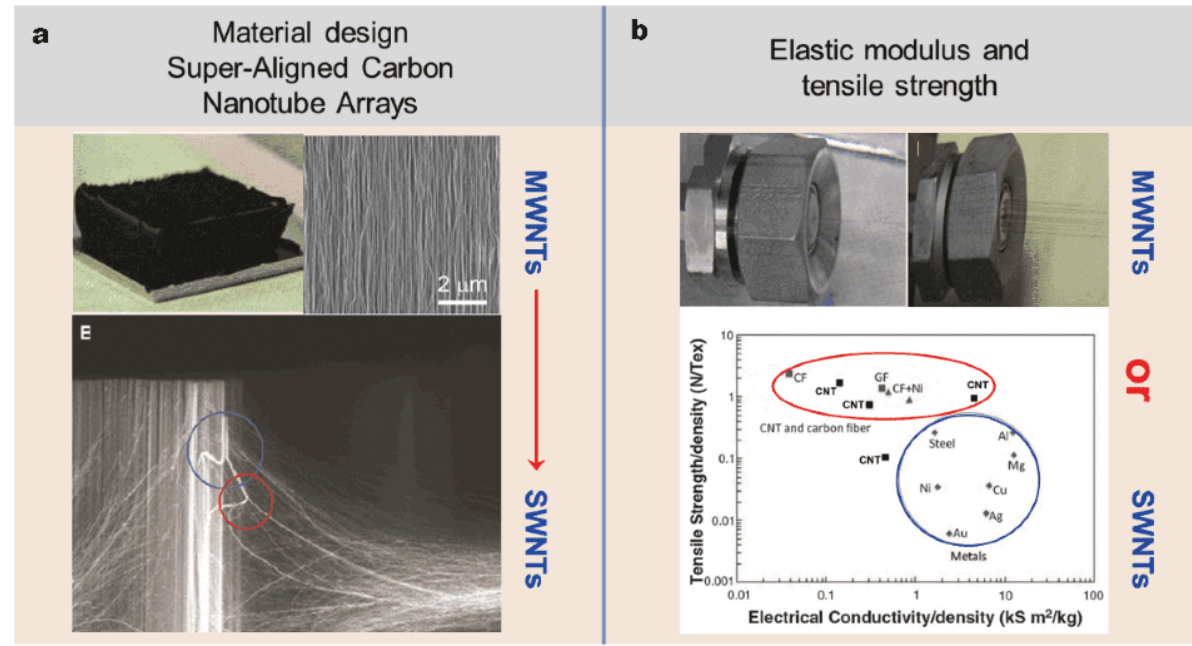

Figure 3 Applications based on controlling of CNT diameter or number of walls: material design (a) (Reprinted with permission from Ref. [59], Copyright 2004, American Association for the Advancement of Science (AAAS); [83], Copyright 2011, American Chemical Society) and increasing the strength of materials (b) (Reprinted with permission from Ref. [84], Copyright 2013, AAAS). The red circle in (b) represents the CNTs and carbon fibers and the blue circle represents other metal materials. 
properties, metallic and semiconducting. In most applications based on the electrical properties, only CNT samples with uniform conductivity (metallic or semiconducting) can meet the requirements. Most of the MWNTs are metallic [25]. The raw SWNTs are usually obtained in mixture with $1 / 3$ metallic and $2 / 3$ semiconducting ones. To improve the purity of SWNTs, many methods have been developed, mainly concentrating on three types of methods, including the direct growth method [86-95], post-treatment [90-95] and solution separation [96-98].

\section{Direct control in CVD system and post-treatment}

Because of the higher chemical reactivity of metallic SWNTs than semiconducting ones, researchers have tried to treat the SWNT mixture with appropriate etchants (oxygen, OH radical, etc.) [88,89], or to apply external irradiation/field with high energy (plasma, UV-light, xenon irradiation, microwave, etc.) [93-95] to enrich semiconducting nanotubes. Some s-SWNTs with small diameters could also be removed because the activity of SWNT is also affected by the diameter difference. Fig. 4a shows the current progress on post-treatment and directgrowth methods. In these methods, strategies based on chemical reactivity, mainly gas etching, usually offer a relative low selectivity than those based on electrical properties. Up to now, the most efficient method to enrich s-SWNTs is electrical breakdown and nearly 100 percent $\mathrm{m}$-SWNTs can be removed with all the s-SWNTs retained [99]. However, this method is not suitable for the high density samples like more than 100 nanotubes $/ \mu \mathrm{m}$. Obviously, the big disadvantage of post-treatment method is the degradation of array density, since at least $1 / 3$ of the as-grown SWNTs are removed. Therefore, improving the density of CNTs during CVD growth is very important.

For direct growth method, it can provide CNTs with high density but relatively low selectivity. Although much
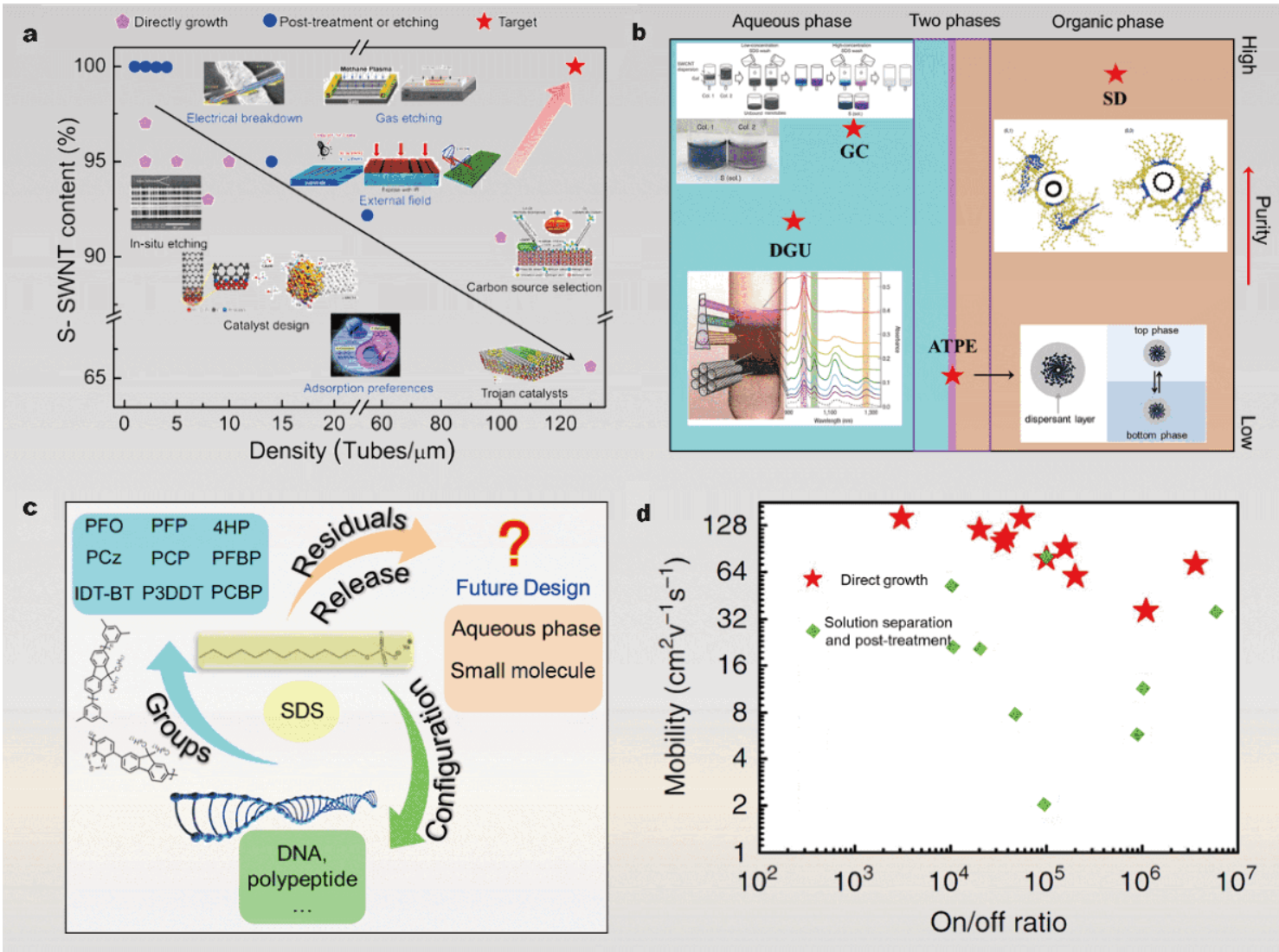

Figure 4 Separation of metallic and semiconducting CNTs. (a) The ideal and real situation of the CNT horizontal array on substrate. (b) High-purity CNTs through solution-based separation methods: DGU, GC, APTE and SD. These methods are developed in different phases. (c) The developing trends of solution-based separation strategy, including groups and configuration designing, and the future designing. (d) Comparison between the CNTs devices fabricated by direct growth and solution-based separation (Reprinted with permission from Ref. [104], Copyright 2016, Springer Nature). 
effort has been made by adopting mixed carbon source, bimetal catalysts, etc., the best selectivity is still much lower than $99 \%$. The main reason is that the two types of nanotubes show very little difference during their growth process. Considering that the electrical properties of SWNTs uniquely depend on the chiral index, chirality control based on the catalysts design shows great possibility to overcome the limitation of density and purity simultaneously. For example, using WC catalyst, semiconducting $(8,4)$ nanotubes could be enriched with about $80 \%$ selectivity and the density exceeds 10 tubes/ $\mu \mathrm{m}$ [70]. On the other hand, an electro-renucleation approach recently has been developed to twist the chirality of the CNTs, and nearly defect-free semiconducting CNT horizontal array with less than $0.1 \%$ residual metallic CNTs has been obtained on the substrate [100].

In terms of purity and density, post treatment and direct growth methods fall far behind the target of 99.9999\% semiconducting selectivity and high density of more than 125 nanotubes $/ \mu \mathrm{m}$ in horizontal array [101]. However, the advantages of these methods are the clean surfaces of SWNTs as well as the easily controlled orientation on substrates. To solve the purity and density, direct growth method combined with chirality control with electric field, could finally make a breakthrough.

\section{Solution separation}

Solution-phase separation method attracts more and more attention because of its high efficiency and the outstanding performance of the final products. This method mainly includes four routes: density gradient ultracentrifugation (DGU) [96], gel chromatography (GC) [97], aqueous two phases extraction (ATPE) [98] and selective dispersion (SD) $[102,103]$, as summarized in Fig. $4 \mathrm{~b}$. These strategies have been developed to enhance the purity of SWNTs with specific properties. For example, the purity of either m- or s-SWNTs has been proved to be higher than $99 \%$, even $99.9 \%$ for s-SWNTs. In DGU method, density gradient medium and the adopted surfactants are the key factors affecting the separation efficiency. Even SWNTs with single chirality can be obtained through introducing the appropriate surfactants in the nonlinear density gradient medium. Similar to DGU, the separation efficiency of GC also depends on the surfactants and stationary phase. Besides, temperature and $\mathrm{pH}$ can affect the separation results as well. However, the high technical threshold and relatively low separation efficiency limit the further improvement of these methods. Interestingly, ATPE utilizes the different distributions of CNTs with different structures in two immiscible aqueous phases formed by the addition of two polymers. This method has the advantages of short separation time, low cost, high yield and high concentration of SWNTs, which is believed to be the most promising large-scale separation method. However, this method relies on the strict control of polymerization degree of each polymer, and its purity is not high enough for further utilization. SD method using biomolecules [103] or conjugated polymers [102] can get the highest purity in solutionseparation methods, but it is limited by the high cost of biomolecules and species of conjugated polymers. Moreover, the removal of polymers is still a great challenge. Although DGU and GC have been applied to the commercial separation, a method to separate SWNTs in large scale with low cost is still desired.

On the other hand, the introduction of defects by ultrasonication and the wrapping of surfactants around SWNTs significantly decrease the performance of device. For example, the mobility of these nanotubes is usually lower than $300 \mathrm{~cm}^{2} \mathrm{~V}^{-1} \mathrm{~s}^{-1}$, mostly resulted from the energy barriers for electron transfer between molecules and nanotubes. To overcome these disadvantages of liquidphase separation in the future, both quality and quantity should be considered and the key lies in the molecular selection and design. As shown in Fig. 4c, from SDS, DNA, to PFO, molecular design has undergone changes from configuration to the functional group. In the future, designing new small molecules with similar active side chains or fragments of conjugated polymers which can release CNTs to water seems to be a promising strategy to improve the quantity and quality.

\section{Applications based on electrical properties}

The successful separation or selective growth of $\mathrm{m}$ - and sSWNTs will become a revolutionary process in many important applications. Because of high carrier mobility and low scattering rate, high quality s-SWNTs attract much attention for fabricating transistors. As shown in Fig. 4d [104], it is obvious that solution-phase separation could provide samples with enough selectivity but degrade their intrinsic electrical properties. This means that different transistors (Fig. 5a) [5,33], like FET and TFT, should be fabricated by using s-SWNTs obtained from different methods. The as-grown CNTs are better for fabricating FETs, which usually demand CNT array with much higher mobility. Since the first CNT transistor was reported in 1998 [30], several milestones have been created, including the first SWNT tunneling FET with a subthreshold swing of $<60 \mathrm{mV} /$ decade in 2004 [31], SWNT FETs with sub-10-nm channel lengths in 2012 


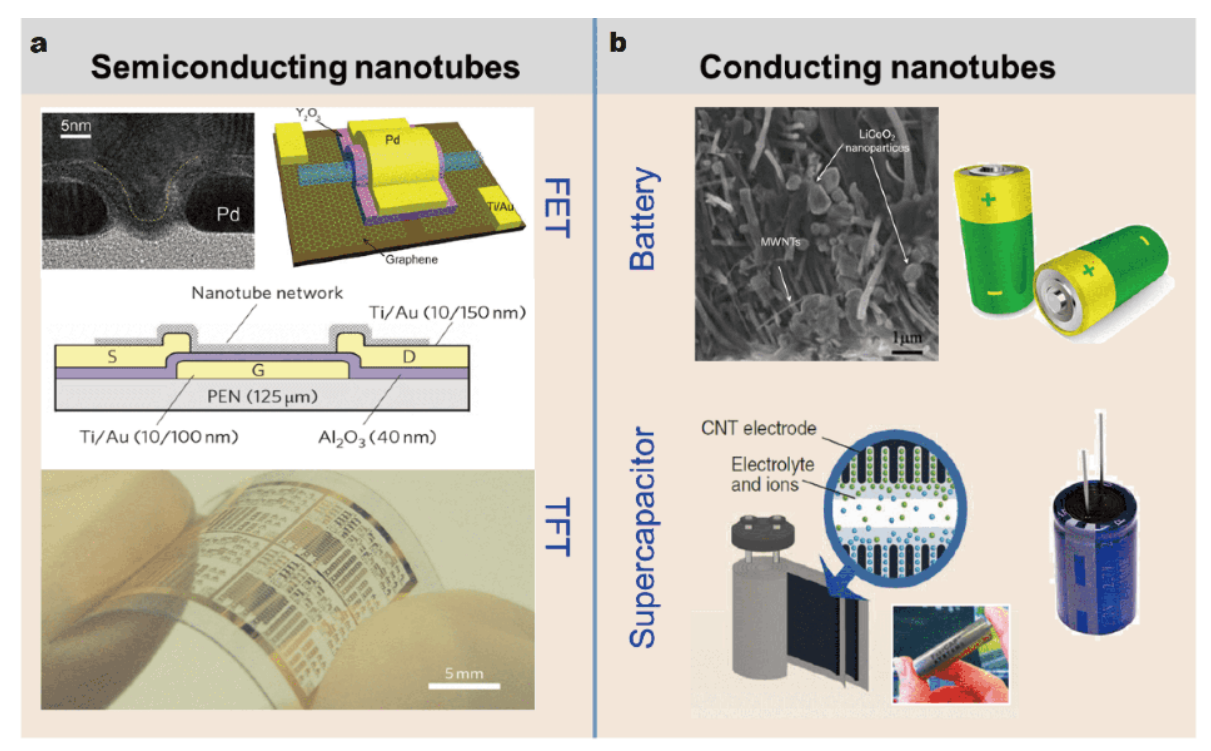

Figure 5 Different applications based on conducting and semiconducting nanotubes. (a) Semiconducting nanotubes are used for fabricating FET and TFT with high mobility and on/off ratio (Reprinted with permission from Ref. [5], Copyright 2017, AAAS; [33], Copyright 2011, Springer Nature). (b) Metallic nanotubes are applied in energy storage field, such as battery and supercapacitor (Reprinted with permission from Ref. [14], Copyright 2013, AAAS; [107], Copyright 2008, Wiley-VCH).

[32] and the first CNT computer in 2013 [105]. Afterwards, CNT complementary transistors with 5-nm gate lengths were realized in 2017 [5]. In 2018, a DS-FET with a CNT channel was reported [106], which can work three times faster than 14-nm commercial silicon transistor while consumes only a quarter of its energy. All of these prove that CNT FETs exhibit better performance than silicon transistors.

For TFTs, hundreds of mobility are enough and CNT film can be deposited by solution method at low temperature and nonvacuum. More importantly, CNTs can be deposited on any substrate with various shapes as expected through solution method. In 2011, the flexible CNT TFTs (Fig. 5a), showing a mobility of $35 \mathrm{~cm}^{2} \mathrm{~V}^{-1} \mathrm{~s}^{-1}$ and a high on/off ratio of $6 \times 10^{6}$, were demonstrated [33]. If further improving the CNT surface deposited from solution and removing the polymers residual, the performance of TFT must be greatly improved.

Besides s-SWNTs, metallic CNTs can also be used in many fields, such as battery and supercapacitor (Fig. 5b) $[14,107]$. Metallic CNTs, mainly MWNTs, have been widely used in lithium ion batteries as the cathodes and anodes through mixing with active materials and polymer binders, such as $1 \mathrm{wt} \% \mathrm{CNTs}$ loading in $\mathrm{LiCoO}_{2}$ [107]. MWNTs can increase the electrical conductivity of cathodes to improve the transmission rate of electrons in the battery and the mechanical strength of batteries. At present, lithium ion batteries with MWNTs have made a major commercial success. Many publications have reported that CNTs could be fabricated as electrodes for supercapacitors to present much higher power capabilities and storage capacities [14,108,109]. Electrodes made from SWNTs and MWNTs gave capacitances of 180 and $102 \mathrm{~F} \mathrm{~g} \mathrm{~g}^{-1}$, respectively [14,108,109]. And, the recent results indicate that an energy density of $16 \mathrm{~W} \mathrm{~h} \mathrm{~kg}^{-1}$ and a power density of $10 \mathrm{~kW} \mathrm{~kg}^{-1}$ were obtained with a maximum voltage of $3.5 \mathrm{~V}$ for a supercapacitor by using forest-grown SWNTs as electrode but without any binder and additive [14]. This is attractive and the performance could be further improved by replacing SWNT bundles and MWNTs with unbundled SWNTs.

\section{Aggregate synthesis decides application dimensions}

As mentioned above, CNTs possess outstanding properties, which decide their wide applications in mechanical field, electronic filed and so on. However, the nanostructure of an individual CNT limits its application. It is necessary to bridge the nano- and macro- structure of CNTs, which can broaden the application dimensions of CNTs and the synthesis of different aggregation morphologies is the key point. Studies found that hierarchical structures are contributed to the new properties of mass transport, shape recovery, terahertz polarization, 
and thermoacoustic sound for certain devices by taking the CNTs as basic unit [110]. According to the macroscopic morphology of CNTs aggregates, they can be roughly divided into three categories: 1D structures, like CNT ropes [111] and fibers [112]; 2D structures, like CNT films [48,113], horizontal arrays [77] and Bucky papers [114]; and 3D structures, like CNT cotton [115], vertically arrays [116], sponges [20] and gels [117-119]. Representative examples of the three aggregate statuses are demonstrated in Fig. 6 and the details are discussed as follows.

CNT aggregation of $1 \mathrm{D}$ structure mainly refers to CNT fibers or yarns. For fabricating fibers, CVD method is dominated and the fibers can be obtained either through direct CVD synthesis or spinning from other CNT aggregates. Two basic methods for large-scale production of CNT fibers are "dry spinning" and "wet spinning" [120]. In dry-spinning method, fibers can be obtained through direct spinning of CNT aerogel $[121,122]$ or from a vertical CNT arrays called "CNT forest" $[18,123,124]$. Wet spinning method means that the fibers are formed from a starting solution through cooling, solvent removal or electrical field [120]. The advantage of this solution method is to make composite fibers by merging other functional materials with CNTs, such as polymers and biomolecules $[125,126]$. The 1D structure of CNT fibers decides their unique application fields. They provide strengthening and flexibility for composite materials and advanced functions. CNTs and their composite fibers can be weaved into textiles [127]. The mechanical properties and electrical conductivities of CNT fibers promote their application in strain sensors [128], electrical wirings [129] and biologic field [130]. In addition, CNT fibers are also considered as the potential materials in aviation, automobiles, energy, and defense industries in the near future [112].

CNT aggregation of 2D structure includes different kinds of CNT films. These 2D films usually consist of random or aligned CNTs on substrate and some can be freestanding like Bucky Papers [114,131]. CVD method is still the basic process for fabricating CNT films. Films on substrates can be grown directly by loading catalyst [77] on the substrates or through floating-catalyst CVD system [132]. Others can be prepared by dissolving and solution filtering from CNT powders [133]. Many publications reported the various solution-based deposition methods, such as Langmuir-Blodgett [134], self-assembly [135], dip coating [136], Meyer rod coating [137], and electrophoretic deposition [138], to prepare 2D CNT films. It should be mentioned that the forces between

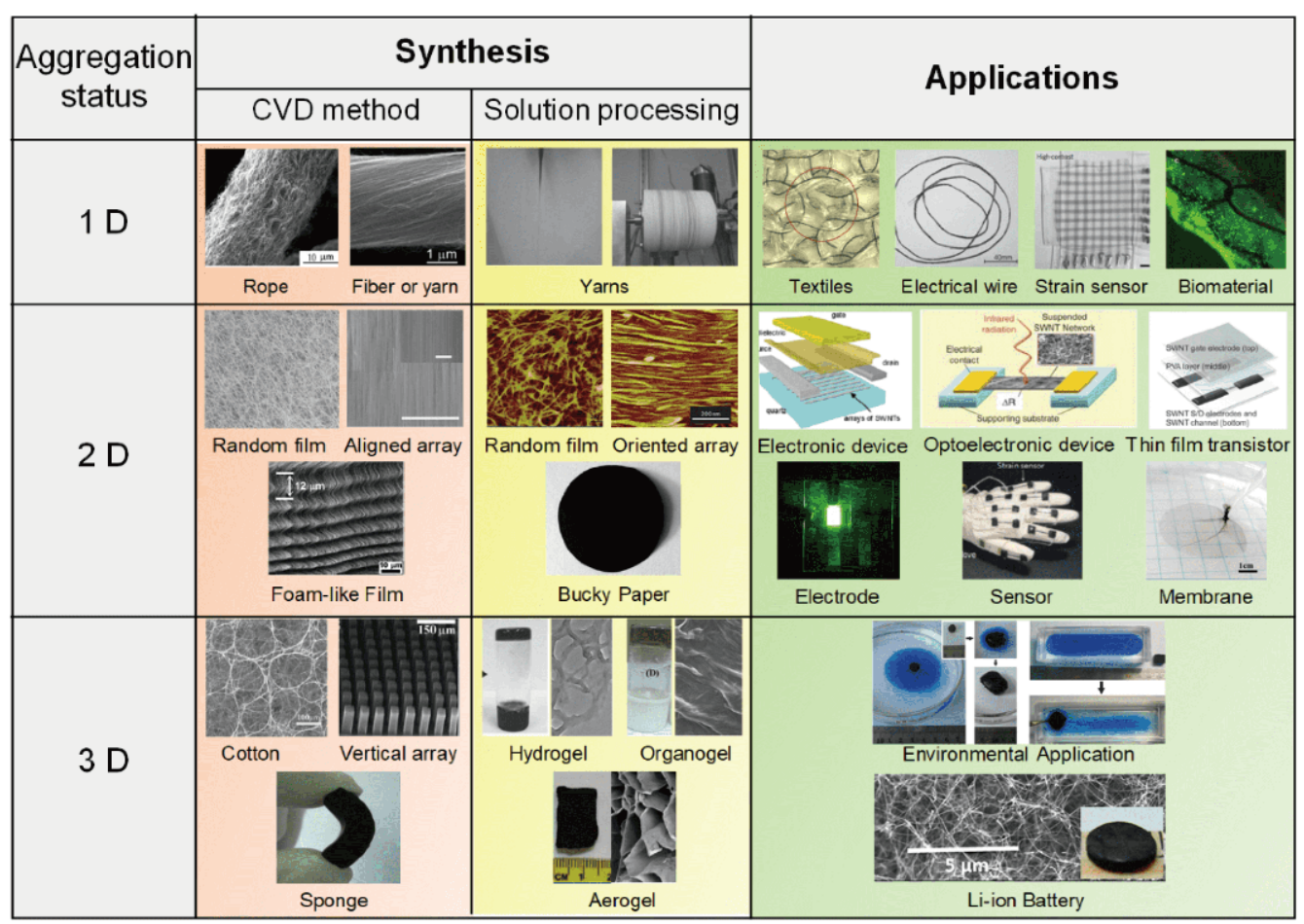

Figure 6 The syntheses and wide applications of CNTs with different aggregation statuses. 
dispersion agents (polymers, surfactants, DNA, etc.) in solution and the CNT bundles are double-edged swords, because the removal of dispersion agents is also a key question in the post-treatment process. 2D CNT films are the most widely used CNT aggregation status. CNT microelectronic and optoelectronic devices with excellent performances have been demonstrated. In macroscopic aspect, CNT films can be used to fabricate TFT [139] and transparent electrodes [140,141]. More applications like sensors [142], wearable devices [143], polarizers [144] and membranes [145], have also been fulfilled based on different alignment and properties of CNT films.

CNT aggregation of $3 \mathrm{D}$ structure refers to the architectures with the increased amount of CNTs per projected unit compared with 2D aggregation. They are macroscopic, engineered products based on assembled CNTs with controlled orientation or configuration [110]. This concept of hierarchical 3D organization can offer some additional properties and enhance the performances, as an important step towards practical applications. CVD techniques have been used to prepare vertically aligned CNT arrays [146], cottons [115] and macroscopic sponge [20]. Other 3D structures like CNT hydrogel [117], organogel [147] and aerogel [148,149] can be obtained from CNT suspensions with the help of polymers, surfactants and so on. The light weight, high porosity, and large surface area of CNT-based 3D products make them promising for environmental applications such as sorption, filtration and separation [20,150]. Composites with synergetic materials can be used as high-performance cathodes in Li-ion batteries [151].

For further improving the quality and quantity of different CNT aggregations, it is guided to know that CNT production process not only depends on molecular reaction, but also involves multiphase reactor, hydrodynamics, gas dispersion, mass or heat transfer, and system engineering [152]. Therefore, advanced knowledge is needed on how the yield, purity and morphology of aggregates are controlled by the parameters in the kinetic process, including size and distribution of catalysts, growth time, feedstocks and operation window. In addition, intratubular van der Waals interactions, external magnetic or electric fields should be taken into account to manipulate the orientation of CNTs.

\section{Scale-up technology decides future industrialization}

Facing the increasing requirements towards practical application, scale-up synthesis of CNT has attracted more and more attention not only in research institution, but also in industrial manufacture. Scale-up synthesis should guarantee the supply of materials of large scale with low cost. Compared with arc discharge and laser ablation, CVD technology has the advantages of relatively mild operating conditions and controllable synthesis, so it is the most promising method for the scale-up of CNTs [153]. However, scale up of CNTs is a very complex process containing continuous production, mass transfer and post-processing, which is far from those studied in fundamental research. Therefore, different equipment with novel methods and engineering principles are needed. Three typical scale-up methods of floating-catalyst CVD (FCCVD), fluidized bed reactor and vertical array technique have been developed to prepare large quantity of CNTs with different macroscopic morphologies.

In the FCCVD method, the catalyst is driven into the hot reaction zone from a syringe filled with catalyst dispersions or by the vaporization of catalyst at a proper temperature. The catalysts used in this system are usually organometallic compounds, especially metallocenes and metal-CO complexes, typically ferrocene and $\mathrm{Fe}(\mathrm{CO})_{5}$ $[152,154]$. FCCVD is a manageable and cost-effective strategy for the production of CNT aerogels [155]. Furthermore, the as-prepared CNTs can be directly deposited on a membrane or continuously be transferred onto other substrates like PET [156]. Compared with the solution filtering technique to deposit films, FCCVD method is much simpler, and has less contamination and CNT damages. The FCCVD equipment can be divided into vertical architecture and horizontal architecture (Fig. 7a). Through FCCVD method, a SWCNT thin film with a length of more than $2 \mathrm{~m}$ has been obtained on a flexible PET substrate [157], and many large-scale, flexible, and transparent electronics have been reported $[132,158]$. The challenge for FCCVD is how to control the wall number, diameter, length, bundling, and $\mathrm{s}-/ \mathrm{m}$ - ratio of CNTs. Therefore, the optimization of equipment, design of catalyst and intensive knowledge of CNT hydromechanics may be helpful.

Fluidized-bed (FB) CVD technique has been developed for large-scale production of CNT powders, due to its advantages of uniform mass and heat transfer, sufficient growth space, and continuous operation [153,159]. This process introduces an input gas to achieve fluidization of catalyst particles. Although it is difficult to fluidize individual CNTs in such a reactor [160], the strong interactions between CNTs lead to the formation of large-size aggregation, which can then be fluidized. Aligned CNTs can also be produced using fluidized CVD by tuning the catalyst structure. For example, CNT arrays can be grown among the layers of vermiculite in a fluidized bed reactor 

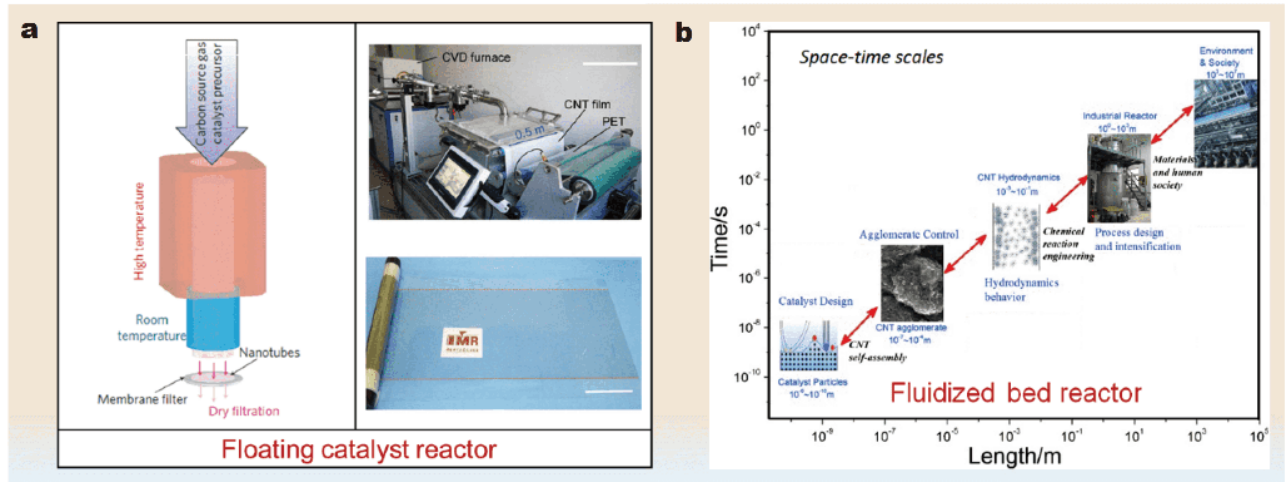

Figure 7 Scale-up synthesis of CNTs through CVD methods: (a) floating catalyst method (Reprinted with permission from Ref. [33], Copyright 2011, Springer Nature; [157], Copyright 2018, Wiley-VCH) and (b) fluidized bed reactors (Reprinted with permission from Ref. [153]. Copyright 2011, Wiley-VCH). For both two methods, catalyst efficiency and equipment design should be considered for different demands.

[161]. This method can reach a high yield, but the purity and quality are relatively low comparing with other methods. A five-level analysis on the mass production of CNT powders has been put forward (Fig. 7b), including the CNT assembly at the atomic scale, CNT agglomerate structure, transport phenomena and growth kinetics, process operation in industrial reactor, and finally the environmental and ecological considerations [153]. Based on this analysis, agglomerated CNTs have been successfully scaled up on the ton scale, and it is available in the market.

Vertically aligned CNT (VACNT) arrays can also be produced in large scale. In 2003, Hata et al. [59] in AIST developed a "super-growth" method to grow SWNT vertical arrays with high yield. Later this technology was developed as a roll-to-roll method for SWNT growth. For large-scale production of VACNTs, flat [162] and curved surfaces [163] were used, and then a strategy of intercalating VACNTs into layered compounds was proposed [164]. This method can directly construct a layered hybrid nanocomposite composed of alternate CNT films and inorganic sheets. Furthermore, it is also effective in fluidized-bed reactor and can realize the mass production.

Though scale-up production has been developed rapidly, there are still several problems limiting its further industrialization and application. Comparing with SWNT growth in laboratory, the selective growth in scale-up production is still difficult to be achieved. Though most SWNT products are reported to show large-chirality enrichment, the controllable synthesis is still in an immature state. Unfortunately, some of the requirements seem to be paradox, which makes it difficult to make a breakthrough in this area. For example, to realize a high quality of SWNT, a high growth temperature is necessary, while the catalysts trend to melt and lose the ability to control SWNT structure at that high temperature. Another big problem is the purity of SWNT, especially the content of metal impurity, for the metal residue could significantly influence the yield and performance of SWNT-based device. This requirement is clearly reflected on the price of SWNT products, for the cost of SWNT with metal impurity less than $1 \%$ is usually 4 times higher than that with metal impurity less than $3.5 \%$. Besides, the production capacity and cost also need to be optimized. So far, FBCVD method could offer SWNT capacity of $1000 \mathrm{~kg}$ per year with the price lower than $\$ 2000 / \mathrm{kg}$ [165]. Overall, a kind of metal-free catalyst with high melting point, high efficiency and uniform structure should be helpful to break through the technical barrier. Besides, a high ability to crack carbon source under lower temperature may decrease the production temperature, which could be more environmentally friendly and economically.

\section{SUMMARY AND OUTLOOK}

CNTs have shown many potential applications in many different areas, especially transparent conductors, nanodevices and thermal interfaces. However, the development of CNT products today is still limited by the immature synthesis technology, which causes structure defects, inhomogeneity, degradation of properties in macroscale, and high cost. To overcome these problems, several aspects should be considered simultaneously.

To improve the performance of individual CNTs and CNT samples from microscale, controlling the fine 


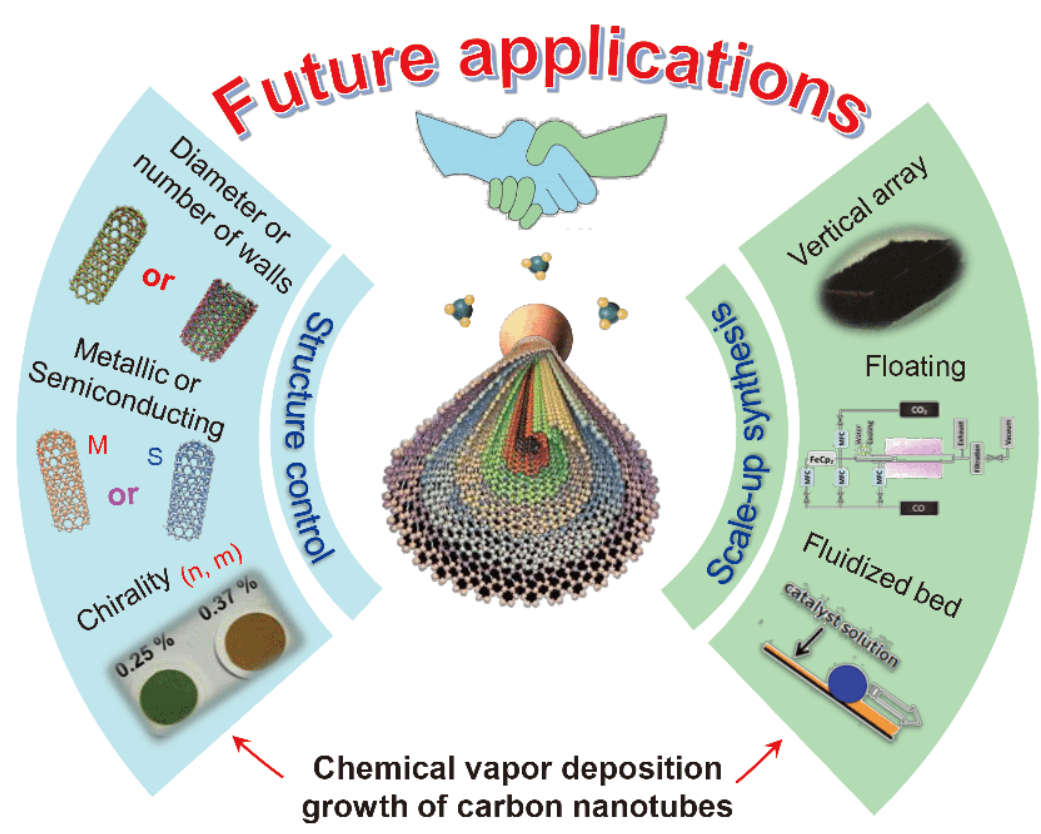

Figure 8 Future design for CVD growth of CNTs in industrialization. Fine structure control should be combined with scale-up synthesis, and this will determine the killer applications of CNTs.

structure of CNTs, especially of SWNTs, is the key. Although many efforts have been devoted to controlling the diameter, electrical properties and even chirality of SWNTs, it is still not enough. From the view of theoretical point, further understanding on the detailed growth mechanism is still needed, for example, how CNT cap forms in atomic scale, what's the fine structure between CNT and catalyst, and how the growth model affects the chirality preference of SWNTs. Experimentally, more methods to grow SWNTs with uniform structure should be further developed and the catalyst design, including high-temperature tolerance and the epitaxial growth from SWNT segments should be focused on.

On the other hand, the scale-up synthesis of SWNTs should also be paid attention to. Since cost-effective CNTs are more competitive to increase the quality as well as the quantity, simplifying the operation process between production and application in order to avoid destroying the perfect structure of CNTs as little as possible and synthesizing CNTs via high purity with metal-free catalyst are welcome. Moreover, as the bridge between the microscopic properties of individual CNTs and macroscale performance of CNT productions, the aggregation status should be designed particularly. Direct synthesis of CNT productions with proper aggregates is an attractive project.

Meanwhile, it is not enough to realize the above re- quirements separately. It is the combination that could finally determine the future of CNTs, as shown in Fig. 8. In other words, the combination of structure control and scale-up synthesis, or the cooperation between lab and industry, is the key step for the real application of CNTs. For example, if SWNT segments can be used as catalysts for an efficient epitaxial growth and this technology can be utilized in large-scale growth via $\mathrm{FB}$, the final products should have the advantages of uniform structure, metalfree and high yield, without most of the current difficulties. In a word, there is still a long way to make CNTs become an irreplaceable material in the future. However, the rapid development and continuous attention give us the sufficient confidence. We believe CNT can show its excellent performance and make our life colorful in the future.

Received 19 July 2019; accepted 7 August 2019;

published online 9 September 2019

1 Iijima S. Helical microtubules of graphitic carbon. Nature, 1991, 354: $56-58$

2 Iijima S, Ichihashi T. Single-shell carbon nanotubes of 1-nm diameter. Nature, 1993, 363: 603-605

3 Cao Q, Rogers JA. Ultrathin films of single-walled carbon nanotubes for electronics and sensors: A review of fundamental and applied aspects. Adv Mater, 2009, 21: 29-53

4 Ionescu AM, Riel H. Tunnel field-effect transistors as energyefficient electronic switches. Nature, 2011, 479: 329-337 
5 Qiu C, Zhang Z, Xiao M, et al. Scaling carbon nanotube complementary transistors to 5-nm gate lengths. Science, 2017, 355: 271-276

6 Avouris P, Freitag M, Perebeinos V. Carbon-nanotube photonics and optoelectronics. Nat Photon, 2008, 2: 341-350

7 He X, Hartmann NF, Ma X, et al. Tunable room-temperature single-photon emission at telecom wavelengths from $\mathrm{sp}^{3}$ defects in carbon nanotubes. Nat Photon, 2017, 11: 577-582

8 Shi Z, Hong X, Bechtel HA, et al. Observation of a Luttingerliquid plasmon in metallic single-walled carbon nanotubes. Nat Photon, 2015, 9: 515-519

9 Avouris P, Appenzeller J, Martel R, et al. Carbon nanotube electronics. Proc IEEE, 2003, 9: 1772-1784

10 Peng B, Locascio M, Zapol P, et al. Measurements of near-ultimate strength for multiwalled carbon nanotubes and irradiationinduced crosslinking improvements. Nat Nanotech, 2008, 3: 626631

11 Jin SH, Dunham SN, Song J, et al. Using nanoscale thermocapillary flows to create arrays of purely semiconducting singlewalled carbon nanotubes. Nat Nanotech, 2013, 8: 347-355

12 Gong K, Du F, Xia Z, et al. Nitrogen-doped carbon nanotube arrays with high electrocatalytic activity for oxygen reduction. Science, 2009, 323: 760-764

13 Baughman RH, Zakhidov AA, de Heer WA. Carbon nanotubesthe route toward applications. Science, 2002, 297: 787-792

14 De Volder MF, Tawfick SH, Baughman RH, Hart AJ. Carbon nanotubes: present and future commercial applications. Science, 2013, 339: 535-539

15 Chen Y, Zhang J. Chemical vapor deposition growth of singlewalled carbon nanotubes with controlled structures for nanodevice applications. Acc Chem Res, 2014, 47: 2273-2281

16 Chen Y, Zhang Y, Hu Y, et al. State of the art of single-walled carbon nanotube synthesis on surfaces. Adv Mater, 2014, 26: 5898-5922

17 Zhang R, Zhang Y, Wei F. Controlled synthesis of ultralong carbon nanotubes with perfect structures and extraordinary properties. Acc Chem Res, 2017, 50: 179-189

18 Zhang X, Jiang K, Feng C, et al. Spinning and processing continuous yarns from 4-inch wafer scale super-aligned carbon nanotube arrays. Adv Mater, 2006, 18: 1505-1510

19 Wang Y, Wei F, Luo G, et al. The large-scale production of carbon nanotubes in a nano-agglomerate fluidized-bed reactor. Chem Phys Lett, 2002, 364: 568-572

20 Gui X, Wei J, Wang K, et al. Carbon nanotube sponges. Adv Mater, 2010, 22: 617-621

21 Penev ES, Artyukhov VI, Yakobson BI. Extensive energy landscape sampling of nanotube end-caps reveals no chiral-angle bias for their nucleation. ACS Nano, 2014, 8: 1899-1906

22 Yao Y, Feng C, Zhang J, et al. "Cloning” of single-walled carbon nanotubes via open-end growth mechanism. Nano Lett, 2009, 9: 1673-1677

23 Zhang R, Zhang Y, Zhang Q, et al. Growth of half-meter long carbon nanotubes based on Schulz-Flory distribution. ACS Nano, 2013, 7: 6156-6161

24 Dresselhaus MS, Dresselhaus G, Saito R. Physics of carbon nanotubes. Carbon, 1995, 33: 883-891

25 Wei BQ, Vajtai R, Ajayan PM. Reliability and current carrying capacity of carbon nanotubes. Appl Phys Lett, 2001, 79: 11721174

26 Chou TW, Gao L, Thostenson ET, et al. An assessment of the science and technology of carbon nanotube-based fibers and composites. Compos Sci Tech, 2010, 70: 1-19

27 Franklin AD. Nanomaterials in transistors: From high-performance to thin-film applications. Science, 2015, 349: aab2750

28 Skákalová V, Dettlaff-Weglikowska U, Roth S. Electrical and mechanical properties of nanocomposites of single wall carbon nanotubes with PMMA. Synth Met, 2005, 152: 349-352

29 Koerner H, Liu W, Alexander M, et al. Deformation-morphology correlations in electrically conductive carbon nanotube-thermoplastic polyurethane nanocomposites. Polymer, 2005, 46: 4405-4420

30 Tans SJ, Verschueren ARM, Dekker C. Room-temperature transistor based on a single carbon nanotube. Nature, 1998, 393: 4952

31 Appenzeller J, Lin YM, Knoch J, et al. Band-to-band tunneling in carbon nanotube field-effect transistors. Phys Rev Lett, 2004, 93 : 196805

32 Franklin $\mathrm{AD}$, Luisier M, Han SJ, et al. Sub-10 nm carbon nanotube transistor. Nano Lett, 2012, 12: 758-762

33 Sun DM, Timmermans MY, Tian Y, et al. Flexible high-performance carbon nanotube integrated circuits. Nat Nanotech, 2011, 6: $156-161$

34 LeMieux MC, Roberts M, Barman S, et al. Self-sorted, aligned nanotube networks for thin-film transistors. Science, 2008, 321: 101-104

35 Kim P, Shi L, Majumdar A, et al. Thermal transport measurements of individual multiwalled nanotubes. Phys Rev Lett, 2001, 87: 215502

36 Balandin AA, Ghosh S, Bao W, et al. Superior thermal conductivity of single-layer graphene. Nano Lett, 2008, 8: 902-907

37 Han Z, Fina A. Thermal conductivity of carbon nanotubes and their polymer nanocomposites: A review. Prog Polym Sci, 2011, 36: 914-944

38 Mazov IN, Ilinykh IA, Kuznetsov VL, et al. Thermal conductivity of polypropylene-based composites with multiwall carbon nanotubes with different diameter and morphology. J Alloys Compd, 2014, 586: S440-S442

39 Biercuk MJ, Llaguno MC, Radosavljevic M, et al. Carbon nanotube composites for thermal management. Appl Phys Lett, 2002, 80: 2767-2769

40 Kam NWS, O'Connell M, Wisdom JA, et al. Carbon nanotubes as multifunctional biological transporters and near-infrared agents for selective cancer cell destruction. Proc Natl Acad Sci USA, 2005, 102: 11600-11605

41 de la Zerda A, Zavaleta C, Keren S, et al. Carbon nanotubes as photoacoustic molecular imaging agents in living mice. Nat Nanotech, 2008, 3: 557-562

42 Heller DA, Baik S, Eurell TE, et al. Single-walled carbon nanotube spectroscopy in live cells: Towards long-term labels and optical sensors. Adv Mater, 2005, 17: 2793-2799

43 Gao G, Vecitis CD. Electrochemical carbon nanotube filter oxidative performance as a function of surface chemistry. Environ Sci Technol, 2011, 45: 9726-9734

44 Rahaman MS, Vecitis CD, Elimelech M. Electrochemical carbonnanotube filter performance toward virus removal and inactivation in the presence of natural organic matter. Environ Sci Technol, 2012, 46: 1556-1564

45 Corry B. Designing carbon nanotube membranes for efficient water desalination. J Phys Chem B, 2008, 112: 1427-1434

46 Charlier JC. Defects in carbon nanotubes. Acc Chem Res, 2002, 
35: 1063-1069

47 Li X, Zhang L, Wang X, et al. Langmuir-Blodgett assembly of densely aligned single-walled carbon nanotubes from bulk materials. J Am Chem Soc, 2007, 129: 4890-4891

48 Cao Q, Han SJ, Tulevski GS, et al. Arrays of single-walled carbon nanotubes with full surface coverage for high-performance electronics. Nat Nanotech, 2013, 8: 180-186

49 Wang C, Zhang J, Ryu K, et al. Wafer-scale fabrication of separated carbon nanotube thin-film transistors for display applications. Nano Lett, 2009, 9: 4285-4291

50 Koziol K, Vilatela J, Moisala A, et al. High-performance carbon nanotube fiber. Science, 2007, 318: 1892-1895

51 Wu Z, Chen Z, Du X, et al. Transparent, conductive carbon nanotube films. Science, 2004, 305: 1273-1276

$52 \mathrm{Xu}$ YQ, Flor E, Kim MJ, et al. Vertical array growth of small diameter single-walled carbon nanotubes. J Am Chem Soc, 2006, 128: 6560-6561

53 Cheung CL, Kurtz A, Park H, et al. Diameter-controlled synthesis of carbon nanotubes. J Phys Chem B, 2002, 106: 2429-2433

54 He M, Magnin Y, Amara H, et al. Linking growth mode to lengths of single-walled carbon nanotubes. Carbon, 2017, 113: 231-236

55 Gohier A, Ewels CP, Minea TM, et al. Carbon nanotube growth mechanism switches from tip- to base-growth with decreasing catalyst particle size. Carbon, 2008, 46: 1331-1338

56 Wirth CT, Bayer BC, Gamalski AD, et al. The phase of iron catalyst nanoparticles during carbon nanotube growth. Chem Mater, 2012, 24: 4633-4640

57 He M, Jiang H, Kauppinen EI, et al. Diameter and chiral angle distribution dependencies on the carbon precursors in surfacegrown single-walled carbon nanotubes. Nanoscale, 2012, 4: 73947398

58 Tian Y, Timmermans MY, Kivistö S, et al. Tailoring the diameter of single-walled carbon nanotubes for optical applications. Nano Res, 2011, 4: 807-815

59 Hata K, Futaba DN, Mizuno K, et al. Water-assisted highly efficient synthesis of impurity-free single-walled carbon nanotubes. Science, 2004, 306: 1362-1364

60 Hayashi T, Kim YA, Matoba T, et al. Smallest freestanding singlewalled carbon nanotube. Nano Lett, 2003, 3: 887-889

61 Li Y, Kim W, Zhang Y, et al. Growth of single-walled carbon nanotubes from discrete catalytic nanoparticles of various sizes. J Phys Chem B, 2001, 105: 11424-11431

62 An L, Owens JM, McNeil LE, et al. Synthesis of nearly uniform single-walled carbon nanotubes using identical metal-containing molecular nanoclusters as catalysts. J Am Chem Soc, 2002, 124: 13688-13689

63 Kukovitsky EF, L'vov SG, Sainov NA. VLS-growth of carbon nanotubes from the vapor. Chem Phys Lett, 2000, 317: 65-70

64 Kang L, Hu Y, Liu L, et al. Growth of close-packed semiconducting single-walled carbon nanotube arrays using oxygendeficient $\mathrm{TiO}_{2}$ nanoparticles as catalysts. Nano Lett, 2015, 15 : 403-409

65 Yang F, Wang X, Zhang D, et al. Chirality-specific growth of single-walled carbon nanotubes on solid alloy catalysts. Nature, 2014, 510: 522-524

66 Zhang S, Tong $\mathrm{L}, \mathrm{Hu} \mathrm{Y}$, et al. Diameter-specific growth of semiconducting SWNT arrays using uniform $\mathrm{Mo}_{2} \mathrm{C}$ solid catalyst. J Am Chem Soc, 2015, 137: 8904-8907

67 Huang S, Woodson M, Smalley RE, et al. Growth mechanism of oriented long single walled carbon nanotubes using "fast-heating" chemical vapor deposition process. Nano Lett, 2004, 4: 1025-1028

68 Smalley RE, Li Y, Moore VC, et al. Single wall carbon nanotube amplification: En route to a type-specific growth mechanism. J Am Chem Soc, 2006, 128: 15824-15829

69 Liu J, Wang C, Tu X, et al. Chirality-controlled synthesis of single-wall carbon nanotubes using vapour-phase epitaxy. Nat Commun, 2012, 3: 1199

70 Zhang S, Kang L, Wang X, et al. Arrays of horizontal carbon nanotubes of controlled chirality grown using designed catalysts. Nature, 2017, 543: 234-238

71 Zhang S, Tong L, Zhang J. The road to chirality-specific growth of single-walled carbon nanotubes. Natl Sci Rev, 2017, 5: 310-312

72 Zhang S, Wang X, Yao F, et al. Controllable growth of $(n, n-1)$ family of semiconducting carbon nanotubes. Chem, 2019, 5: 1182-1193

73 Magnin Y, Amara H, Ducastelle F, et al. Entropy-driven stability of chiral single-walled carbon nanotubes. Science, 2018, 362: 212215

74 Ding F, Harutyunyan AR, Yakobson BI. Dislocation theory of chirality-controlled nanotube growth. Proc Natl Acad Sci USA, 2009, 106: 2506-2509

75 Artyukhov VI, Penev ES, Yakobson BI. Why nanotubes grow chiral. Nat Commun, 2014, 5: 4892

76 Yuan Q, Xu Z, Yakobson BI, et al. Efficient defect healing in catalytic carbon nanotube growth. Phys Rev Lett, 2012, 108: 245505

$77 \mathrm{Hu}$ Y, Kang L, Zhao Q, et al. Growth of high-density horizontally aligned SWNT arrays using Trojan catalysts. Nat Commun, 2015, 6: 6099

78 Bedewy M, Meshot ER, Guo H, et al. Collective mechanism for the evolution and self-termination of vertically aligned carbon nanotube growth. J Phys Chem C, 2009, 113: 20576-20582

79 Terrones M, Ajayan PM, Banhart F, et al. N-doping and coalescence of carbon nanotubes: Synthesis and electronic properties. Appl Phys A-Mater Sci Processing, 2002, 74: 355-361

80 Goyanes S, Rubiolo GR, Salazar A, et al. Carboxylation treatment of multiwalled carbon nanotubes monitored by infrared and ultraviolet spectroscopies and scanning probe microscopy. Diamond Related Mater, 2007, 16: 412-417

81 Kosynkin DV, Higginbotham AL, Sinitskii A, et al. Longitudinal unzipping of carbon nanotubes to form graphene nanoribbons. Nature, 2009, 458: 872-876

82 Gojny FH, Wichmann MHG, Köpke U, et al. Carbon nanotubereinforced epoxy-composites: Enhanced stiffness and fracture toughness at low nanotube content. Compos Sci Tech, 2004, 64: 2363-2371

83 Kuznetsov AA, Fonseca AF, Baughman RH, et al. Structural model for dry-drawing of sheets and yarns from carbon nanotube forests. ACS Nano, 2011, 5: 985-993

84 Behabtu N, Young CC, Tsentalovich DE, et al. Strong, light, multifunctional fibers of carbon nanotubes with ultrahigh conductivity. Science, 2013, 339: 182-186

85 Bai Y, Zhang R, Ye X, et al. Carbon nanotube bundles with tensile strength over $80 \mathrm{GPa}$. Nat Nanotech, 2018, 13: 589-595

86 Ding L, Tselev A, Wang J, et al. Selective growth of well-aligned semiconducting single-walled carbon nanotubes. Nano Lett, 2009, 9: 800-805

87 Che Y, Wang C, Liu J, et al. Selective synthesis and device applications of semiconducting single-walled carbon nanotubes 
using isopropyl alcohol as feedstock. ACS Nano, 2012, 6: 74547462

88 Kang L, Zhang S, Li Q, et al. Growth of horizontal semiconducting SWNT arrays with density higher than 100 tubes/ $\mu \mathrm{m}$ using ethanol/methane chemical vapor deposition. J Am Chem Soc, 2016, 138: 6727-6730

89 Zhang S, Hu Y, Wu J, et al. Selective scission of $\mathrm{C}-\mathrm{O}$ and $\mathrm{C}-\mathrm{C}$ bonds in ethanol using bimetal catalysts for the preferential growth of semiconducting SWNT arrays. J Am Chem Soc, 2015, 137: 1012-1015

90 Hong G, Zhou M, Zhang R, et al. Separation of metallic and semiconducting single-walled carbon nanotube arrays by "scotch tape". Angew Chem Int Ed, 2011, 50: 6819-6823

91 Du F, Felts JR, Xie X, et al. Laser-induced nanoscale thermocapillary flow for purification of aligned arrays of single-walled carbon nanotubes. ACS Nano, 2014, 8: 12641-12649

$92 \mathrm{Hu}$ Y, Chen Y, Li P, et al. Sorting out semiconducting singlewalled carbon nanotube arrays by washing off metallic tubes using SDS aqueous solution. Small, 2013, 9: 1306-1311

93 Zhang G, Qi P, Wang X, et al. Selective etching of metallic carbon nanotubes by gas-phase reaction. Science, 2006, 314: 974-977

94 Maehashi K, Ohno Y, Inoue K, et al. Chirality selection of singlewalled carbon nanotubes by laser resonance chirality selection method. Appl Phys Lett, 2004, 85: 858-860

95 Hong G, Zhang B, Peng B, et al. Direct growth of semiconducting single-walled carbon nanotube array. J Am Chem Soc, 2009, 131: 14642-14643

96 Arnold MS, Green AA, Hulvat JF, et al. Sorting carbon nanotubes by electronic structure using density differentiation. Nat Nanotech, 2006, 1: 60-65

97 Liu H, Nishide D, Tanaka $\mathrm{T}$, et al. Large-scale single-chirality separation of single-wall carbon nanotubes by simple gel chromatography. Nat Commun, 2011, 2: 309

98 Khripin CY, Fagan JA, Zheng M. Spontaneous partition of carbon nanotubes in polymer-modified aqueous phases. J Am Chem Soc, 2013, 135: 6822-6825

99 Collins PG, Arnold MS, Avouris P. Engineering carbon nanotubes and nanotube circuits using electrical breakdown. Science, 2001, 292: 706-709

100 Wang J, Jin X, Liu Z, et al. Growing highly pure semiconducting carbon nanotubes by electrotwisting the helicity. Nat Catal, 2018, 1: 326-331

101 Franklin AD. The road to carbon nanotube transistors. Nature, 2013, 498: 443-444

102 Nish A, Hwang JY, Doig J, et al. Highly selective dispersion of single-walled carbon nanotubes using aromatic polymers. Nat Nanotech, 2007, 2: 640-646

103 Zheng M, Jagota A, Semke ED, et al. DNA-assisted dispersion and separation of carbon nanotubes. Nat Mater, 2003, 2: 338-342

104 Zhang F, Hou PX, Liu C, et al. Growth of semiconducting singlewall carbon nanotubes with a narrow band-gap distribution. Nat Commun, 2016, 7: 11160

105 Shulaker MM, Hills G, Patil N, et al. Carbon nanotube computer. Nature, 2013, 501: 526-530

106 Qiu C, Liu F, Xu L, et al. Dirac-source field-effect transistors as energy-efficient, high-performance electronic switches. Science, 2018, 361: 387-392

107 Sotowa C, Origi G, Takeuchi M, et al. The reinforcing effect of combined carbon nanotubes and acetylene blacks on the positive electrode of lithium-ion batteries. ChemSusChem, 2008, 1: 911-
915

108 An KH, Kim WS, Park YS, et al. Electrochemical properties of high-power supercapacitors using single-walled carbon nanotube electrodes. Adv Funct Mater, 2001, 11: 387-392

109 Niu C, Sichel EK, Hoch R, et al. High power electrochemical capacitors based on carbon nanotube electrodes. Appl Phys Lett, 1997, 70: 1480-1482

110 Nardecchia S, Carriazo D, Ferrer ML, et al. Three dimensional macroporous architectures and aerogels built of carbon nanotubes and/or graphene: Synthesis and applications. Chem Soc Rev, 2013, 42: 794-830

111 Zhang X, Cao A, Li Y, et al. Self-organized arrays of carbon nanotube ropes. Chem Phys Lett, 2002, 351: 183-188

112 Zhang X, Li Q, Holesinger T, et al. Ultrastrong, stiff, and lightweight carbon-nanotube fibers. Adv Mater, 2007, 19: 4198-4201

113 Li J, Hu L, Wang L, et al. Organic light-emitting diodes having carbon nanotube anodes. Nano Lett, 2006, 6: 2472-2477

114 Li Z, Xu J, O'Byrne JP, et al. Freestanding bucky paper with high strength from multi-wall carbon nanotubes. Mater Chem Phys, 2012, 135: 921-927

115 Zheng L, Zhang X, Li Q, et al. Carbon-nanotube cotton for largescale fibers. Adv Mater, 2007, 19: 2567-2570

116 Fan S, Chapline MG, Franklin NR, et al. Self-oriented regular arrays of carbon nanotubes and their field emission properties. Science, 1999, 283: 512-514

117 Du R, Wu J, Chen L, et al. Hierarchical hydrogen bonds directed multi-functional carbon nanotube-based supramolecular hydrogels. Small, 2014, 10: 1387-1393

118 Pal A, Chhikara BS, Govindaraj A, et al. Synthesis and properties of novel nanocomposites made of single-walled carbon nanotubes and low molecular mass organogels and their thermo-responsive behavior triggered by near IR radiation. J Mater Chem, 2008, 18: 2593-2600

119 Zou J, Liu J, Karakoti AS, et al. Ultralight multiwalled carbon nanotube aerogel. ACS Nano, 2010, 4: 7293-7302

120 Yadav MD, Dasgupta K, Patwardhan AW, et al. High performance fibers from carbon nanotubes: Synthesis, characterization, and applications in composites-a review. Ind Eng Chem Res, 2017, 56: 12407-12437

121 Reguero V, Alemán B, Mas B, et al. Controlling carbon nanotube type in macroscopic fibers synthesized by the direct spinning process. Chem Mater, 2014, 26: 3550-3557

122 Motta M, Moisala A, Kinloch I, et al. High performance fibres from 'dog bone' carbon nanotubes. Adv Mater, 2007, 19: 37213726

123 Li Q, Zhang X, DePaula R, et al. Sustained growth of ultralong carbon nanotube arrays for fiber spinning. Adv Mater, 2006, 18: 3160-3163

124 Alvarez NT, Miller P, Haase M, et al. Carbon nanotube assembly at near-industrial natural-fiber spinning rates. Carbon, 2015, 86: 350-357

125 Barisci J, Tahhan M, Wallace G, et al. Properties of carbon nanotube fibers spun from DNA-stabilized dispersions. Adv Funct Mater, 2004, 14: 133-138

126 Razal J, Gilmore K, Wallace G. Carbon nanotube biofiber formation in a polymer-free coagulation bath. Adv Funct Mater, 2008, 18: 61-66

127 Zhang M, Atkinson KR, Baughman RH. Multifunctional carbon nanotube yarns by downsizing an ancient technology. Science, 2004, 306: 1358-1361 
Zhao H, Zhang Y, Bradford PD, et al. Carbon nanotube yarn strain sensors. Nanotechnology, 2010, 21: 305502

129 Lekawa-Raus A, Patmore J, Kurzepa L, et al. Electrical properties of carbon nanotube based fibers and their future use in electrical wiring. Adv Funct Mater, 2014, 24: 3661-3682

130 Edwards SL, Church JS, Werkmeister JA, et al. Tubular microscale multiwalled carbon nanotube-based scaffolds for tissue engineering. Biomaterials, 2009, 30: 1725-1731

131 Kakade BA, Pillai VK, Late DJ, et al. High current density, low threshold field emission from functionalized carbon nanotube bucky paper. Appl Phys Lett, 2010, 97: 073102

132 Jiang S, Hou PX, Chen ML, et al. Ultrahigh-performance transparent conductive films of carbon-welded isolated single-wall carbon nanotubes. Sci Adv, 2018, 4: eaap9264

133 Yu LP, Shearer C, Shapter J. Recent development of carbon nanotube transparent conductive films. Chem Rev, 2016, 116: 13413-13453

134 Kim Y, Minami N, Zhu W, et al. Langmuir-Blodgett films of single-wall carbon nanotubes: Layer-by-layer deposition and inplane orientation of tubes. Jpn J Appl Phys, 2003, 42: 7629-7634

135 El-Aguizy T, Kim SG. Large-scale assembly of carbon nanotubes. ASME 2004 3rd Integrated Nanosystems Conference, Pasadena, USA, 2004, 97-98

136 Saran N, Parikh K, Suh DS, et al. Fabrication and characterization of thin films of single-walled carbon nanotube bundles on flexible plastic substrates. J Am Chem Soc, 2004, 126: 4462-4463

$137 \mathrm{Hu} \mathrm{L}$, Gruner G, Jenkins J, et al. Flash dry deposition of nanoscale material thin films. J Mater Chem, 2009, 19: 5845-5849

138 Kamat PV, Thomas KG, Barazzouk S, et al. Self-assembled linear bundles of single wall carbon nanotubes and their alignment and deposition as a film in a DC field. J Am Chem Soc, 2004, 126: 10757-10762

139 Park S, Pitner G, Giri G, et al. Large-area assembly of densely aligned single-walled carbon nanotubes using solution shearing and their application to field-effect transistors. Adv Mater, 2015, 27: 2656-2662

140 Hussain A, Liao Y, Zhang Q, et al. Floating catalyst CVD synthesis of single walled carbon nanotubes from ethylene for high performance transparent electrodes. Nanoscale, 2018, 10: 9752-9759

141 Feng C, Liu K, Wu JS, et al. Flexible, stretchable, transparent conducting films made from superaligned carbon nanotubes. Adv Funct Mater, 2010, 20: 885-891

142 Snow ES, Perkins FK, Houser EJ, et al. Chemical detection with a single-walled carbon nanotube capacitor. Science, 2005, 307: 1942-1945

143 Zhou J, Xu X, Yu H, et al. Deformable and wearable carbon nanotube microwire-based sensors for ultrasensitive monitoring of strain, pressure and torsion. Nanoscale, 2017, 9: 604-612

144 Ren L, Pint CL, Booshehri LG, et al. Carbon nanotube terahertz polarizer. Nano Lett, 2009, 9: 2610-2613

145 Lee B, Baek Y, Lee M, et al. A carbon nanotube wall membrane for water treatment. Nat Commun, 2015, 6: 7109

146 Cho W, Schulz M, Shanov V. Growth and characterization of vertically aligned centimeter long CNT arrays. Carbon, 2014, 72: 264-273

147 Chen J, Xue C, Ramasubramaniam R, et al. A new method for the preparation of stable carbon nanotube organogels. Carbon, 2006, 44: $2142-2146$

148 Kohlmeyer RR, Lor M, Deng J, et al. Preparation of stable carbon nanotube aerogels with high electrical conductivity and porosity. Carbon, 2011, 49: 2352-2361

149 Bryning M, Milkie D, Islam M, et al. Carbon nanotube aerogels. Adv Mater, 2007, 19: 661-664

150 Gui X, Cao A, Wei J, et al. Soft, highly conductive nanotube sponges and composites with controlled compressibility. ACS Nano, 2010, 4: 2320-2326

151 Chen X, Zhu H, Chen YC, et al. MWCNT/ $\mathrm{V}_{2} \mathrm{O}_{5}$ core/shell sponge for high areal capacity and power density $\mathrm{Li}$-ion cathodes. ACS Nano, 2012, 6: 7948-7955

152 Zhang Q, Huang JQ, Qian WZ, et al. The road for nanomaterials industry: A review of carbon nanotube production, post-treatment, and bulk applications for composites and energy storage. Small, 2013, 9: 1237-1265

153 Zhang Q, Huang JQ, Zhao MQ, et al. Carbon nanotube mass production: Principles and processes. ChemSusChem, 2011, 4: 864-889

154 Moisala A, Nasibulin AG, Brown DP, et al. Single-walled carbon nanotube synthesis using ferrocene and iron pentacarbonyl in a laminar flow reactor. Chem Eng Sci, 2006, 61: 4393-4402

155 Tian Y, Timmermans MY, Partanen M, et al. Growth of singlewalled carbon nanotubes with controlled diameters and lengths by an aerosol method. Carbon, 2011, 49: 4636-4643

156 Laiho P, Mustonen K, Ohno Y, et al. Dry and direct deposition of aerosol-synthesized single-walled carbon nanotubes by thermophoresis. ACS Appl Mater Interfaces, 2017, 9: 20738-20747

157 Wang BW, Jiang S, Zhu QB, et al. Continuous fabrication of meter-scale single-wall carbon nanotube films and their use in flexible and transparent integrated circuits. Adv Mater, 2018, 30: 1802057

158 Kaskela A, Nasibulin AG, Timmermans MY, et al. Aerosol-synthesized SWCNT networks with tunable conductivity and transparency by a dry transfer technique. Nano Lett, 2010, 10: 43494355

159 See CH, Harris AT. A review of carbon nanotube synthesis via fluidized-bed chemical vapor deposition. Ind Eng Chem Res, 2007, 46: 997-1012

160 Geldart D. Types of gas fluidization. Powder Tech, 1973, 7: 285292

161 Zhao MQ, Zhang Q, Huang JQ, et al. Layered double hydroxides as catalysts for the efficient growth of high quality single-walled carbon nanotubes in a fluidized bed reactor. Carbon, 2010, 48: 3260-3270

162 Singh C, Shaffer MSP, Koziol KKK, et al. Towards the production of large-scale aligned carbon nanotubes. Chem Phys Lett, 2003, 372: $860-865$

163 Zhang Q, Huang JQ, Zhao MQ, et al. Radial growth of vertically aligned carbon nanotube arrays from ethylene on ceramic spheres. Carbon, 2008, 46: 1152-1158

164 Qian H, Greenhalgh ES, Shaffer MSP, et al. Carbon nanotubebased hierarchical composites: A review. J Mater Chem, 2010, 20: 4751-4762

165 Jia X, Wei F. Advances in production and applications of carbon nanotubes. Top Curr Chem (Z), 2017, 375: 18

Acknowledgements This work was supported by the Ministry of Science and Technology of China (2016YFA0200101 and 2016YFA0200104), the National Natural Science Foundation of China (51432002, 21790052 and 51720105003), Beijing Municipal Science and Technology Planning Project (Z161100002116026), China Postdoctoral 
Science Foundation (8201400852 and 8201400892), and the National Program for Thousand Young Talents of China.

Author contributions Zhang S, Qian L, Zhao Q, Wang Z, Lin D, Liu $\mathrm{W}$, Zhang J conceived the study and organized the manuscript; Zhang J, Zhang S, Qian L, Chen Y contributed to discussion and revised the manuscript.

Conflict of interest The authors declare no conflict of interest.

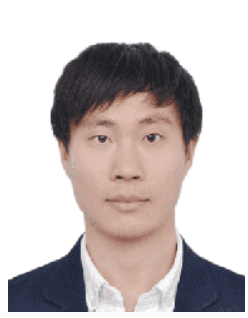

Shuchen Zhang is a postdoctoral researcher at Peking University, China. He received his BSc degree from Shandong University, China, in 2012, and his PhD degree from Peking University. His research interests mainly focus on the preparation and properties of low-dimensional nanomaterials, including the structure-controlled growth of SWNTs and their related applications.

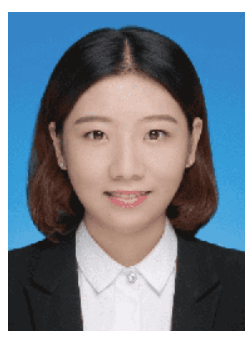

Liu Qian received her $\mathrm{PhD}$ degree (2018) in physical chemistry from the National Center for Nanoscience and Technology, Chinese Academy of Sciences. She then became a postdoctoral research fellow in Prof. Jin Zhang's group in Peking University. Her current research interests focus on the controlled synthesis and optoelectronic applications of CNTs.

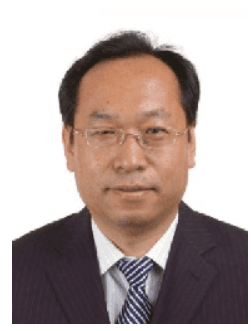

Jin Zhang received his $\mathrm{PhD}$ from Lanzhou University in 1997. After a two-year postdoctoral fellowship at the University of Leeds, UK, he returned to Peking University where he was appointed as Associate Professor (2000) and then promoted to Full Professor in 2006. In 2013, he was appointed as Cheung Kong scholar. He is the Fellow of RSC. His research focuses on the controlled synthesis and spectroscopic characterization of carbon nanomaterials. He has published more than 260 peer-reviewed papers.

\section{碳纳米管: 可控制备决定未来}

张树辰 ${ }^{1}$, 钱柳 ${ }^{1}$, 赵秋辰 ${ }^{1}$, 王泽群 ${ }^{1}$, 林德武 ${ }^{1}$, 刘伟铭 ${ }^{1}$, 陈亚樹 ${ }^{2}$, 张锦 ${ }^{*^{*}}$

摘要 在过去几十年中, 碳纳米管由于其优异的物理和化学性质而 备受关注, 被认为是一个强有力的未来明星材料. 尽管基于碳纳米 管的诸多产品及应用实例相继浮现, 但是碳纳米管所呈现出的实 际性质与理论值之间依然存在较大差异, 无法达到研究者的预期, 这源自于目前尚未成熟的控制制备技术. 碳纳米管的可控制备技 术包括结构的精细控制方法和样品的宏量制备技术, 这在很大程 度上决定了碳纳米管的未来发展前景. 基于此, 本文概述了近几十 年来研究者们在碳纳米管的精细结构控制、聚合状态设计和样品 宏量制备等方面的主要进展, 进一步指出了碳纳米管未来的可控 制备技术必须与特定化的应用紧密结合, 以迎接即将来临的产业 化时代. 